Virginia Commonwealth University vCU Scholars Compass

1999

\title{
Evolution of the electronic structure and properties of neutral and charged aluminum clusters: A comprehensive analysis
}

\author{
B. K. Rao \\ Virginia Commonwealth University \\ P. Jena \\ Virginia Commonwealth University
}

Follow this and additional works at: http://scholarscompass.vcu.edu/phys_pubs

Part of the Physics Commons

Rao, B. K., Jena, P. Evolution of the electronic structure and properties of neutral and charged aluminum clusters: A comprehensive analysis. The Journal of Chemical Physics 111, 1890 (1999). Copyright (C) 1999 AIP Publishing LLC.

\section{Downloaded from}

http://scholarscompass.vcu.edu/phys_pubs/148

This Article is brought to you for free and open access by the Dept. of Physics at VCU Scholars Compass. It has been accepted for inclusion in Physics Publications by an authorized administrator of VCU Scholars Compass. For more information, please contact libcompass@vcu.edu. 


\title{
Evolution of the electronic structure and properties of neutral and charged aluminum clusters: A comprehensive analysis
}

\author{
B. K. Rao and P. Jena \\ Physics Department, Virginia Commonwealth University, Richmond, Virginia 23284-2000
}

(Received 9 March 1999; accepted 3 May 1999)

\begin{abstract}
Density-functional theory with generalized gradient approximation for the exchange-correlation potential has been used to calculate the global equilibrium geometries and electronic structure of neutral, cationic, and anionic aluminum clusters containing up to 15 atoms. The total energies of these clusters are then used to study the evolution of their binding energy, relative stability, fragmentation channels, ionization potential, and vertical and adiabatic electron affinities as a function of size. The geometries are found to undergo a structural change from two dimensional to three dimensional when the cluster contains 6 atoms. An interior atom emerges only when clusters contain 11 or more atoms. The geometrical changes are accompanied by corresponding changes in the coordination number and the electronic structure. The latter is reflected in the relative concentration of the $s$ and $p$ electrons of the highest occupied molecular orbital. Aluminum behaves as a monovalent atom in clusters containing less than seven atoms and as a trivalent atom in clusters containing seven or more atoms. The binding energy evolves monotonically with size, but $\mathrm{Al}_{7}$, $\mathrm{Al}_{7}^{+}, \mathrm{Al}_{7}^{-}, \mathrm{Al}_{11}^{-}$, and $\mathrm{Al}_{13}^{-}$exhibit greater stability than their neighbors. Although the neutral clusters do not conform to the jellium model, the enhanced stability of these charged clusters is demonstrated to be due to the electronic shell closure. The fragmentation proceeds preferably by the ejection of a single atom irrespective of the charge state of the parent clusters. While odd-atom clusters carry a magnetic moment of $1 \mu_{B}$ as expected, clusters containing even number of atoms carry $2 \mu_{B}$ for $n \leqslant 10$ and $0 \mu_{B}$ for $n>10$. The calculated results agree very well with all available experimental data on magnetic properties, ionization potentials, electron affinities, and fragmentation channels. The existence of isomers of $\mathrm{Al}_{13}$ cluster provides a unique perspective on the anomaly in the intensity distribution of the mass spectra. The unusual stability of $\mathrm{Al}_{7}$ in neutral, cationic, and anionic form compared to its neighboring clusters is argued to be due to its likely existence in a mixed-valence state. (C) 1999 American Institute of Physics.
\end{abstract}

[S0021-9606(99)30629-2]

\section{INTRODUCTION}

The development of laser vaporization technique ${ }^{1}$ has enabled experimentalists in the last two decades to produce and characterize atomic clusters of specific size and composition. The considerable interest in this field is brought about by two major expectations. First, atomic clusters form a bridge between molecular physics and condensed matter physics. Thus, by studying the properties of clusters as a function of size, one hopes to learn how the bulk properties evolve. Secondly, atomic clusters may have unique size specific properties that differ from their bulk systems. Consequently, materials synthesized by assembling clusters may be technologically important. In spite of the enormous progress made in this field, prospects for understanding the evolution of properties from clusters to crystals as well as synthesis of cluster assembled materials have remained elusive. The study of the evolution is hampered by the fact that different properties evolve differently and what constitutes bulk behavior is sometimes difficult to define unambiguously. The difficulty in synthesizing cluster assembled materials arises from the fact that (1) size selected clusters cannot be produced in sufficient quantities and (2) that since these are metastable, they would coalesce when brought in the vicinity of each other. However, numerous studies ${ }^{2}$ in the past few years have elucidated a variety of novel properties of atomic clusters brought about by their limited size and dimension. The most studied systems constitute free-electron metals such as the alkalies. Experimentally these are easier to study due to their low melting points. Theoretically the freeelectron nature of these elements permit the use of simple models that can be used to study qualitatively the properties of clusters consisting of even thousands of atoms. Quantitative calculations are, however, limited to clusters containing no more than a couple of dozens of atoms. ${ }^{3}$

Next to the alkalies, aluminum clusters are among the most studied systems. Since, like the alkalies, the electronic structure of bulk aluminum is free-electronlike, it is expected that aluminum clusters can also be studied by applying simple models. The model that has been most successful in describing the electronic structure of alkali metal clusters is the jellium model ${ }^{4}$ where one assumes that the atomic arrangements in the cluster are not very important in describing their electronic structure. One can, thus, approximate the cluster by a spherical distribution of positive ion charge to which the valence electrons respond. Such a description gives rise to electronic shells where the quantum numbers 
order as $1 s^{2}, 1 p^{6}, 1 d^{10}, 2 s^{2}, 1 f^{14}, 2 p^{6} \ldots$ Thus, clusters with $2,8,20,40, \ldots$ electrons can close $1 s^{2}, 1 p^{6}, 2 s^{2}, 2 p^{6} \ldots$ shells, respectively, and in analogy with nuclear shell structure should be very stable. Neutral alkali metal clusters containing 2, 8, 20, 40... atoms, therefore, should exhibit pronounced stability. Similarly positively charged alkali clusters containing $3,9,21,41, \ldots$ atoms should also be very stable. ${ }^{5}$ That this is exactly what was observed experimentally ${ }^{4,6}$ has given tremendous credibility to this simple model.

Several theoretical ${ }^{7-18}$ and experimental ${ }^{19-30}$ studies have been carried out in the last decade to investigate if this simple jellium model is applicable to aluminum clusters. The conclusions concerning the cluster size where the jellium model works have, however, been conflicting. While some experiments suggest that clusters as small as $\mathrm{Al}_{7}$ behave like a jellium, ${ }^{20,30}$ others find the predictions of the jellium model to be at odds with experiment for clusters containing up to 40 atoms. ${ }^{25}$ The major reason lies with the electronic structure of the aluminum atom itself. The atom has a $3 s^{2} 3 p^{1}$ configuration and the $s$ and $p$ orbitals are separated by an energy gap of $4.99 \mathrm{eV}$. In small clusters, one expects the $s$ - $p$ overlap to be small, and thus aluminum may behave as a monovalent atom. It is only when the $s$ - $p$ overlap is large that aluminum can be considered to be trivalent. At what cluster size then does this transition from monovalency to trivalency take place? If aluminum atoms are considered to be monovalent, the magic numbers for neutrals should be for clusters containing $2,8,20,40 \ldots$ atoms and for cations these should be at $3,9,21,41, \ldots$. On the other hand, if aluminum is trivalent, no neutral clusters containing less than 46 atoms can be magic as their valence electrons cannot satisfy the shell closing requirement discussed earlier. It is, however, possible to study electronic shell closure in smaller charged clusters. For example, $\mathrm{Al}_{7}^{+}$and $\mathrm{Al}_{13}^{-}$could be magic as they would contain 20 and 40 valence electrons, respectively (assuming $\mathrm{Al}$ to be trivalent). $\mathrm{Al}_{3}^{+}$should be a magic number since it would contain 2 or 8 electrons depending upon whether $\mathrm{Al}$ is monovalent or trivalent.

The magic numbers can be studied by mass spectroscopy $^{23}$ as well as collision- or photo-induced experiments. $^{20-22}$ The intensity of the peaks in the mass spectra of clusters reflect their stability and an unusually large peak is characteristic of a magic cluster. In fragmentation experiments, magic clusters are often the preferred byproduct. Several experiments of mass ion intensities and fragmentation channels have been carried out, but the results are not always in agreement with each other. While one group $^{23}$ has seen no evidence of conspicuous peaks in the mass spectra at $\mathrm{Al}_{3}^{+}$and $\mathrm{Al}_{7}^{+}$, another group ${ }^{20}$ has reported $\mathrm{Al}_{7}^{+}$as a magic cluster. Similarly, while $\mathrm{Al}_{3}^{+}$has been observed to be a dominant product in photo destruction experiment, ${ }^{21}$ no signature of this is found in collision induced fragmentation. ${ }^{20,21}$

In this paper we examine, critically, the available experimental data in aluminum clusters as a function of cluster size and compare these with our first principles calculations of the equilibrium geometries and total energies of neutral, positively, and negatively charged clusters containing up to 15 atoms. We have studied the evolution of their atomic structure, coordination number, interatomic distance, binding energy, fragmentation channels, electronic structure, magnetic properties, ionization potential, and vertical and adiabatic electron affinities. Comparison of these results with experiment and previous theories provides a unique perspective of the evolution of cluster properties as well as the role of isomers and mixed-valency on their interpretation.

In Sec. II we discuss briefly our theoretical procedure. The results are discussed in Sec. III. Section IV contains a summary of our conclusions.

\section{THEORETICAL PROCEDURE}

Our calculations are carried out by using the density functional theory and generalized gradient approximation (GGA) for the exchange-correlation potential. The atomic functions are represented by a set of Gaussian orbitals. We have used the LanL2DZ basis ${ }^{31}$ where the inner $1 s, 2 s$, and $2 p$ cores of aluminum were frozen. The valence electrons were represented by double zeta quality functions. The accuracy of this approximation has been earlier established ${ }^{32}$ by comparing the results with those based upon all electron calculations. The molecular orbital representing the cluster was constructed by taking a linear combination of atomic orbitals and determining the combination coefficients by solving the Raleigh-Ritz variational equation. At no stage of the calculations did we introduce any other variable parameters. For the computations, we have used GAUSSIAN 94 software $^{33}$ and the generalized gradient approximation (GGA) prescribed by Becke, Perdew, and Wang (referred to as BPW91 in the GAUSSIAN 94 software).

For a given spin multiplicity the geometry optimization was carried out by the method of steepest descent. No symmetry was imposed on the clusters. This amounted to varying 3n-6 independent coordinates for each cluster containing $n$-atoms $(n \geqslant 3)$. The forces at each atomic site were calculated from the gradient of the total energy. The atoms were then moved along the directions of the force to a new location, and the process was repeated until the forces vanished. For computational purposes, this was considered to have been achieved when the maximum force, the root-meansquare force, the maximum displacement of the atoms, and the root-mean-square displacement of the atoms reached threshold values of 0.00045 a.u./Bohr, 0.0003 a.u./Bohr, 0.0018 a.u., and 0.0012 a.u., respectively. To avoid trapping at local minima of the potential energy surface, the positions of the atoms were randomized and the optimization process was again carried through. Since this procedure is enormously time consuming, for certain clusters, we also started from different initial configurations obtained by Lloyd and Johnston $^{34}$ from molecular-dynamics simulation based on empirical potentials as the starting geometries. Significant rearrangement occurred during the optimization procedure and our resultant equilibrium geometries are generally different from those obtained by Lloyd and Johnston. Since one does not know a priori the spin multiplicity of the clusters, the above calculations were repeated for different spin configurations to obtain the lowest energy structure. As neutral aluminum clusters are expected to have low spin multiplici- 
ties, we examined spin multiplicities of 1 and 3 for evenelectron clusters and 2 and 4 for odd-electron clusters.

In this paper we are concerned with the stability and properties of neutral, cationic (single positive charge), and anionic (single negative charge) clusters. Geometry optimization for all these charge states and two different spin multiplicities were carried out for each of the aluminum clusters containing 2 to 15 atoms. In total, this amounted to optimizing $14 \times 2 \times 3=84$ separate geometries. The results of these optimizations are discussed in the following section.

\section{RESULTS AND DISCUSSIONS}

The past experimental studies ${ }^{19-30}$ on aluminum clusters can be broadly divided into three categories depending upon the properties they are designed to investigate. (1) The relative stability of clusters and the existence of magic numbers (i.e., clusters with unusual stability) are probed through quadrupole or time-of-flight mass spectrometry and collision or photo-induced dissociation. (2) The electronic structure and magnetic properties are probed through measurements of ionization potential, electron affinity, dipole polarizability, and magnetic deflection. (3) The chemistry of the clusters are studied through their interaction with reagent molecules. We will comment on all these experiments during the course of our discussion.

A number of theoretical studies ${ }^{7-18}$ have also been carried out by different groups. They range from the simple jellium model ${ }^{7}$ where the cluster geometry is ignored, to a number of models where the geometry explicitly enters into the picture. In this category, the studies include semiempirical molecular orbital calculations, ${ }^{8}$ molecular-dynamics simulation $^{34}$ based on empirical model potentials, quantummolecular dynamics, ${ }^{13-16,18}$ and quantum-mechanical calculations based on quantum-chemical ${ }^{9-11}$ and densityfunctional $^{12-18}$ theories within local density or local spindensity approximations. The most systematic study was done by Jones ${ }^{14}$ who used simulated annealing and local spindensity approximation in the density-functional theory for neutral and cationic clusters containing up to 10 atoms. After the present work was completed we became aware of the recent calculations of Akola et al. ${ }^{15}$ and Ahlrichs and Elliott $^{16}$ who have optimized the geometries of clusters up to 23 atoms and 15 atoms, respectively. Instead of repeating the discussion of earlier theoretical work, we refer the reader to these articles. ${ }^{14-16}$ To our knowledge no systematic calculations of negatively charged aluminum clusters up to $n=15$ are available. In the following we discuss our results on neutral, cationic, and anionic aluminum clusters sequentially.

\section{A. Equilibrium geometries, nearest-neighbor distances, and coordination numbers}

The starting point in any description of cluster properties is their geometrical structure. Unfortunately, there is no experimental technique that can provide direct information on cluster geometry. The clusters are too large for spectroscopic techniques and too small for diffraction techniques to be of much use. Indirect information on cluster geometries can be obtained by studying their reaction with reagent molecules, ${ }^{35}$ but their interpretation is plagued by assumptions and uncer- (a)
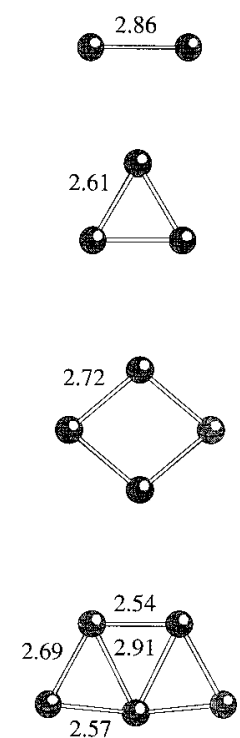

FIG. 1. Ground-state geometries of (a) neutral, (b) cationic, and (c) anionic aluminum clusters containing $2-5$ atoms. Bond lengths are given in $\AA$ units.

tainties. Raman spectroscopy ${ }^{36}$ on matrix isolated clusters has been recently used to study cluster geometries, but the effect of the matrix on the cluster geometry remains a nagging concern.

The only method that enables determination of cluster geometries at present is, thus, based on theoretical calculations. Unfortunately, the calculated geometries depend on the level of the theory. The better the theory, the smaller the size of the cluster it can handle. Simpler theories based on empirical schemes and model potentials can provide geometries of large clusters, but their accuracy remains questionable. The correctness of the geometries determined theoretically can only be established by comparing calculated properties of these clusters with experiment. We believe that we have identified the equilibrium geometries of aluminum clusters containing up to 15 atoms correctly. This belief is based on our ability to explain the mass ion intensity, collision induced fragmentation channels, ionization potentials, vertical and adiabatic electron affinities, and magnetic properties for all clusters studied consistently and quantitatively. We should remind the readers that a cluster can exhibit a multitude of geometrical structures and some of these may lie very close to each other in energy. This has been discussed by Jones $^{14}$ for very small $\mathrm{Al}$ clusters. In order to keep the length of this paper under control, we only discuss the geometries corresponding to the lowest energy states. A discussion of isomers of $\mathrm{Al}_{13}$ and $\mathrm{Al}_{15}$ clusters will be provided later in this paper.

In Figs. 1-3 we present the geometries of neutral and charged (cationic as well as anionic) clusters containing 2-5, 6-10, and 11-15 atoms, respectively. We first discuss the geometries of the neutral clusters. For clusters up to 5 atoms, the ground state structures are planar and are in agreement with the calculations of Jones. ${ }^{14}$ It is worth pointing out that the geometries of neutral clusters for $n \leqslant 5$ are also the same 
(a)

(b)
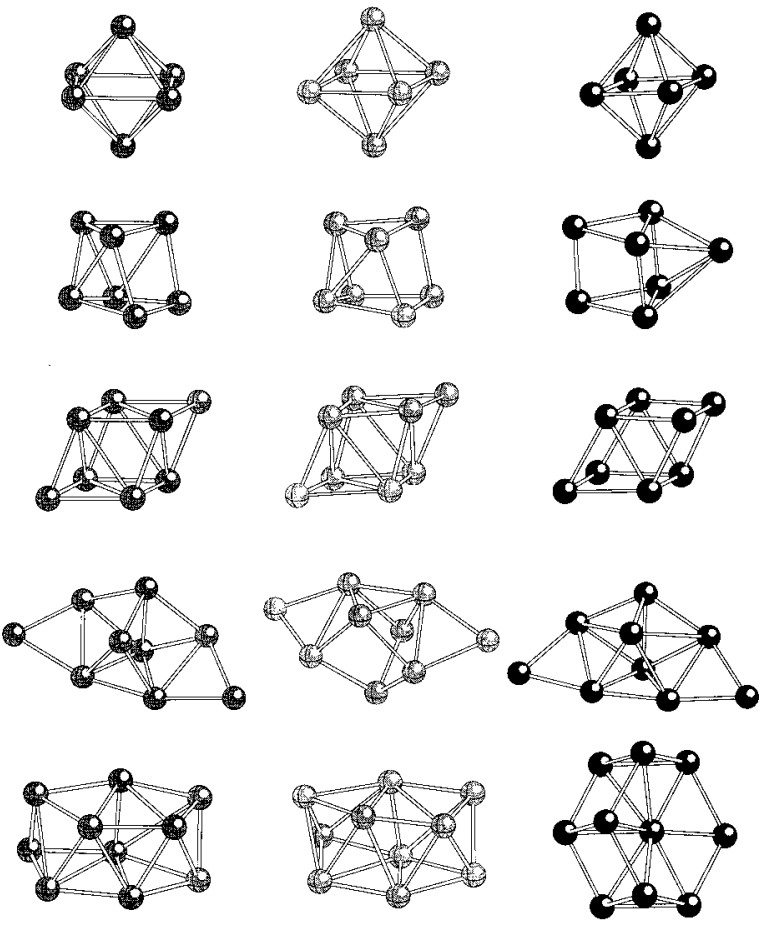

FIG. 2. Ground-state geometries of (a) neutral, (b) cationic, and (c) anionic aluminum clusters containing 6-10 atoms.

as those of the alkali metal clusters. Thus one could interpret these results as being indicative of aluminum clusters existing in a monovalent configuration at least in this size range. We will see later that this is also consistent with the electronic structure of these clusters. For neutral clusters containing 6-10 atoms (Fig. 2), the geometries become three dimensional. $\mathrm{Al}_{9}$ is the smallest cluster that develops a pentagonal arrangement of atoms that is precursor to icosahedric growth. In clusters containing 11-15 atoms (Fig. 3), an interior atom with a bulklike coordination emerges. The ground state of the 13-atom cluster is a Jahn-Teller distorted decahedron where the two pentagons join to form square faces. All earlier calculations had indicated the ground state to be an icosahedron where the two pentagons join to form triangular faces. We have examined the icosahedral arrangement and found the Jahn-Teller distorted icosahedral structure to lie $0.43 \mathrm{eV}$ higher in energy than the decahedral structure. Since no earlier calculations ${ }^{12,13,15-18}$ had predicted the decahedron and none of these calculations were done at the GGA level, we have repeated the calculations using local spin density approximation (LSDA) to see if the difference lies in the choice of the potential. At the LSDA level we still find the decahedron to be lower in energy than the icosahedron, but the difference in energies narrows to $0.3 \mathrm{eV}$. These energy differences are small (namely $0.03 \mathrm{eV} /$ atom at the GGA level) and within the accuracy of our calculations, these two structures can be considered to be nearly degenerate. The 14th atom in $\mathrm{Al}_{14}$ caps one of the square faces and removes the Jahn-Teller distortion of the decahedron otherwise present in the $\mathrm{Al}_{13}$ structure. The $\mathrm{Al}_{15}$ geometry results by capping one of the square faces of the decahedron by two (a)
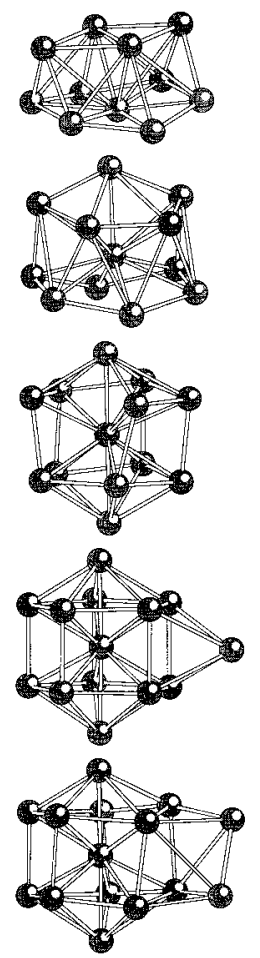

(b)
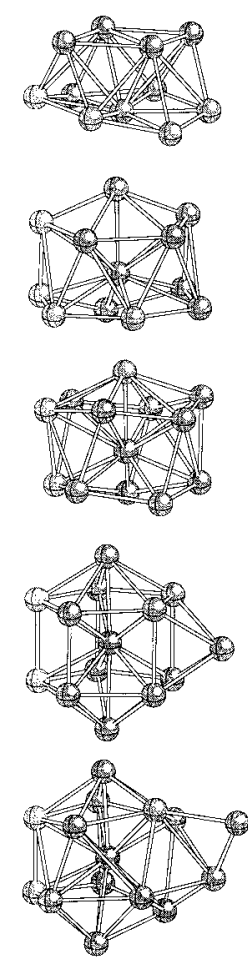

(c)

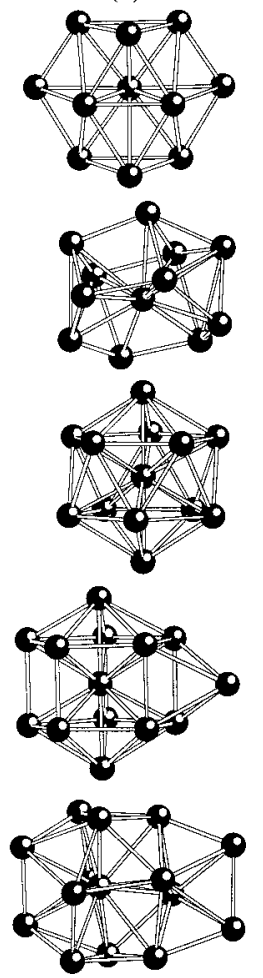

FIG. 3. Ground-state geometries of (a) neutral, (b) cationic, and (c) anionic aluminum clusters containing 11-15 atoms.

atoms. This results in a slight distortion of the decahedron.

Unlike in the bulk where the nearest-neighbor distance is well defined, the lack of a perfect structural symmetry in clusters makes it difficult to assign a unique nearest neighbor distance for each cluster. For example, the nearest-neighbor distance is easy to define in $\mathrm{Al}_{2}, \mathrm{Al}_{3}$, and $\mathrm{Al}_{4}$ as these clusters are symmetric. We have found the ground state of $\mathrm{Al}_{2}$ to be a ${ }^{3} \Pi_{u}$ state with a bond length of $2.86 \AA$. There exists another isomer of ${ }^{3} \Sigma_{g}^{-}$symmetry with a bond length of $2.57 \AA$ with a total energy higher by $0.08 \mathrm{eV}$ only. Several calculations on $\mathrm{Al}_{2}$ are available in the literature where the exchange-correlation potential has been treated within the local spin-density approximations ${ }^{14,15}$ as well as the generalized gradient approximation. ${ }^{16}$ While these authors also find the ${ }^{3} \Pi_{u}$ and the ${ }^{3} \Sigma_{g}^{-}$states (with a long and a short bond, respectively) to be energetically nearly degenerate, they identify ${ }^{3} \Sigma_{g}^{-}$as the ground state. Our result is in agreement with the well established ${ }^{37}$ result that the ground state is ${ }^{3} \Pi_{u}$ with a bond length of $\sim 2.80 \AA$. The binding energy of the dimer obtained from our calculations is $1.43 \mathrm{eV}$ which agrees well with the experimental value ${ }^{38}$ of $1.55 \pm 0.15 \mathrm{eV}$. $\mathrm{Al}_{3}$ is an equilateral triangle and the nearest-neighbor distance is $2.61 \AA$. In $\mathrm{Al}_{4}$ the nearest-neighbor distance is 2.72 $\AA$ and is slightly enlarged over the value in the trimer. However, in $\mathrm{Al}_{5}$ and subsequent clusters the nearest-neighbor distances vary within a narrow range. For example, the " "nearest' -neighbor distances in $\mathrm{Al}_{5}$ range between 2.54 and $2.91 \AA$. We define an atom to be counted as a nearest neighbor if its distance is less than $3.2 \AA$ (which is $12 \%$ larger than the corresponding distance in the bulk). This cutoff was ar- 


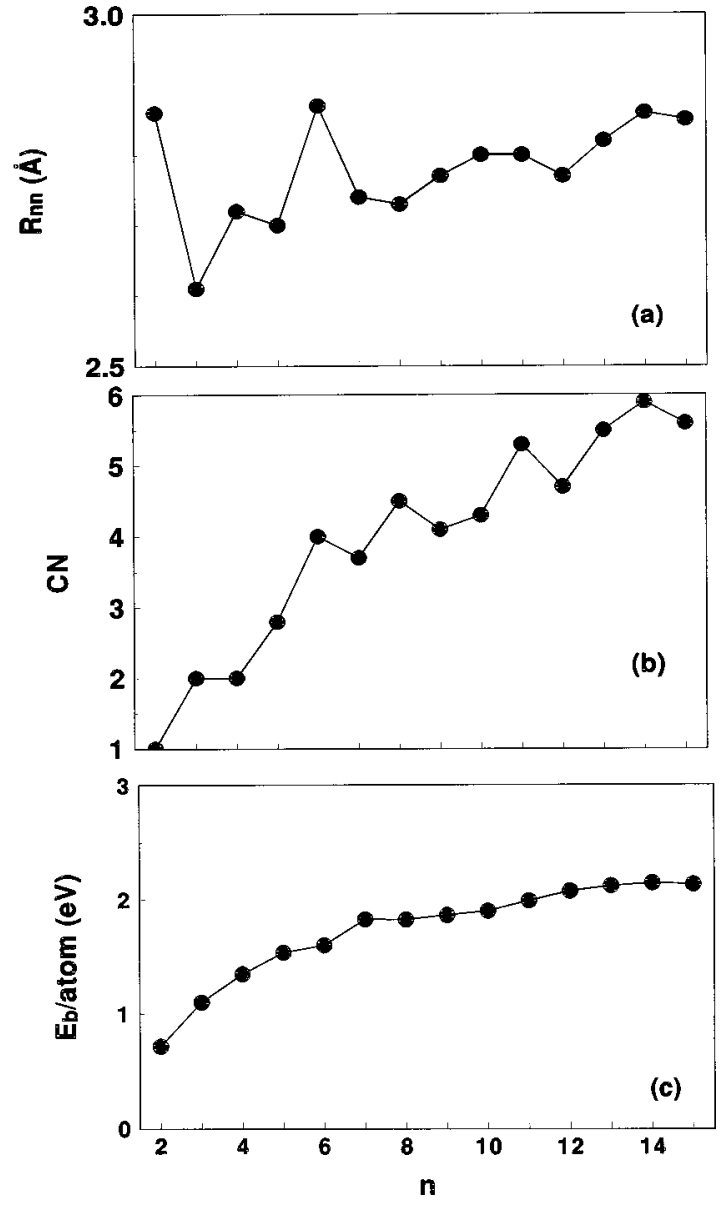

FIG. 4. (a) Average nearest-neighbor distance (b) coordination number, and (c) binding energy/atom as a function of size for neutral $\mathrm{Al}_{n}(n=2-15)$ clusters.

rived at by examining all the interatomic distances in the clusters studied and by noting that there was a distinct gap in these distances beyond $3.2 \AA$. In $\mathrm{Al}_{6}$ to $\mathrm{Al}_{15}$, the nearestneighbor distances lie in the range 2.64-3.1 $\AA, 2.63-2.88 \AA$, 2.68-3.11 $\AA, 2.64-3.03 \AA, 2.68-3.13 \AA, 2.68-3.20 \AA$, 2.62-3.21 $\AA$, 2.71-3.12 $\AA$, 2.65-3.16 , and 2.71-3.15 respectively. In Figs. 1-3 all bonds having lengths of $3.2 \AA$ or less are connected.

In order to study the evolution of the nearest-neighbor distance, we have calculated the average nearest-neighbor distance by using the following expression:

$$
\langle R\rangle=\frac{1}{n_{b}} \sum_{i j} R_{i j} .
$$

Here $R_{i j}$ is the distance between two atoms $i$ and $j$ with a cutoff $=3.2 \AA$ and $n_{b}$ is the total number of bonds between atoms that lie below this cutoff. The results are plotted in Fig. 4(a). Note that with the exception of $\mathrm{Al}_{2}$ and $\mathrm{Al}_{6}$, the average nearest-neighbor distances generally increase with cluster size and reach the bulk limit of $2.86 \AA$ by the time the cluster contains 14 atoms. Thus, one can conclude that the evolution of the nearest-neighbor distance approaches the bulk value rather rapidly.

Unfortunately, the same cannot be said about the geometry. For example, the geometries in Figs. 1-3 do not re-
TABLE I. Average nearest-neighbor distances $(\AA)$ of neutral, cationic, and anionic aluminum clusters.

\begin{tabular}{rccc}
\hline \hline$n$ & Neutral & Cation & Anion \\
\hline 2 & 2.86 & 3.33 & 2.67 \\
3 & 2.61 & 2.83 & 2.69 \\
4 & 2.72 & 2.81 & 2.76 \\
5 & 2.54 & 2.73 & 2.69 \\
6 & 2.64 & 2.90 & 2.83 \\
7 & 2.63 & 2.74 & 2.80 \\
8 & 2.68 & 2.85 & 2.79 \\
9 & 2.65 & 2.77 & 2.76 \\
10 & 2.68 & 2.83 & 2.80 \\
11 & 2.68 & 2.90 & 2.89 \\
12 & 2.62 & 2.82 & 2.84 \\
13 & 2.71 & 2.85 & 2.87 \\
14 & 2.86 & 2.85 & 2.86 \\
15 & 2.85 & 2.85 & 2.86 \\
\hline \hline
\end{tabular}

semble a fragment of the fcc (face-centered-cubic) structure that characterizes bulk aluminum. This can also be viewed by studying the average coordination number. We define this by the following expression:

$$
C N=\frac{1}{n} \sum_{i} N_{i} .
$$

Here $N_{i}$ is the number of nearest-neighbor atoms surrounding the $i$ th atom and the summation is carried over all the atoms with $i$ ranging from 1 to $n$, the total number of atoms. The results are given in Fig. 4(b). Note that there are marked changes in the coordination as a function of size. These occur at $n=6$ and 11 . The coordination numbers are small $(\sim 2)$ for $n<5$ where the geometries are planar. At $n=6$ the geometry becomes three dimensional and the coordination number jumps from 2.8 to 4 . Again, at $n=11$ a bulklike atom appears and the coordination number jumps from 4.3 to 5.3. This can also be seen from Figs. 1-3 by counting the number of bonds which increases from 7 to 12 as one goes from $n$ $=5$ to 6 and from 23 to 31 as one goes from $n=10$ to 11 . In bulk aluminum, the coordination number is 12 and for the atom on the $\langle 111\rangle$ surface it is 9. Thus, the coordination number in the largest cluster studied is far from reaching the surface limit, let alone the bulk limit. This is an indication of the openness of the cluster geometry in spite of the fact that the interatomic distances are bulk like. We will see in the following discussions that these geometry changes affect the electronic structure of the clusters.

As the clusters are charged either by adding an electron or by removing one, their geometries are expected to undergo modifications. In Figs. 1-3 we present the equilibrium geometries of positively and negatively charged aluminum clusters in Figs. 1(b), 1(c), 2(b), 2(c), 3(b), and 3(c), respectively. The average nearest-neighbor distances in charged clusters computed using Eq. (1) are compared with neutral clusters in Table I. We note that these distances are generally larger than those in neutral clusters for $n \leqslant 13$ and become equal in 14- and 15-atom clusters. This is as expected since, in a small cluster, removal or addition of a charge will create a bigger perturbation than in larger clusters. The geometries of charged clusters are generally similar to the neutral clus- 
TABLE II. Total energies and preferred spin multiplicities of neutral, cationic, and anionic clusters of $\mathrm{Al}_{n}$ $(n \leqslant 15)$ in their respective ground-state configurations.

\begin{tabular}{|c|c|c|c|c|c|c|}
\hline \multirow[b]{2}{*}{$n$} & \multicolumn{2}{|c|}{ Neutral } & \multicolumn{2}{|c|}{ Positive ion } & \multicolumn{2}{|c|}{ Negative ion } \\
\hline & $E_{n}$ (hartree) & Multiplicity & $E_{n}^{+}$(hartree) & Multiplicity & $E_{n}^{-}$(hartree) & Multiplicity \\
\hline 1 & -1.93962 & 2 & -1.70919 & 1 & -1.94427 & 3 \\
\hline 2 & -3.93185 & 3 & -3.70957 & 2 & -3.98260 & 4 \\
\hline 3 & -5.94016 & 2 & -5.70480 & 3 & -5.99704 & 3 \\
\hline 4 & -7.95664 & 3 & -7.71775 & 4 & -8.03492 & 2 \\
\hline 5 & -9.98035 & 2 & -9.73767 & 3 & -10.05606 & 1 \\
\hline 6 & -11.99035 & 3 & -11.75329 & 2 & -12.08433 & 2 \\
\hline 7 & -14.04764 & 2 & -13.83183 & 1 & -14.12253 & 1 \\
\hline 8 & -16.05356 & 3 & -15.82941 & 2 & -16.14761 & 2 \\
\hline 9 & -18.07306 & 2 & -17.85456 & 1 & -18.16659 & 1 \\
\hline 10 & -20.09470 & 3 & -19.88158 & 2 & -20.19167 & 2 \\
\hline 11 & -22.14111 & 2 & -21.91244 & 1 & -22.23810 & 1 \\
\hline 12 & -24.19093 & 1 & -23.96728 & 2 & -24.27573 & 2 \\
\hline 13 & -26.22800 & 2 & -26.00601 & 1 & -26.33658 & 1 \\
\hline 14 & -28.25892 & 1 & -28.04741 & 2 & -28.34344 & 2 \\
\hline 15 & -30.27088 & 2 & -30.06953 & 1 & -30.37031 & 1 \\
\hline
\end{tabular}

ters with major exceptions for $n=7,13$, and 15. For example, $\mathrm{Al}_{7}^{-}$geometry is marked by an atom capping a "square" face while $\mathrm{Al}_{7}$ and $\mathrm{Al}_{7}^{+}$structures show no such feature. The geometry of $\mathrm{Al}_{13}^{+}$is significantly different from either an icosahedron or decahedron. The $\mathrm{Al}_{13}^{-}$cluster has two isomers similar to its neutral state: A decahedron and an icosahedron and these do not exhibit Jahn-Teller distortion seen in the neutral geometries. Unlike the neutral $\mathrm{Al}_{13}$ whose decahedral structure lies $0.03 \mathrm{eV} /$ atom lower than the icosahedral one, the two isomers of $\mathrm{Al}_{13}^{-}$are much closer in energy with the decahedral structure being preferred over the icosahedral structure by $0.008 \mathrm{eV} /$ atom. The structure of $\mathrm{Al}_{15}^{-}$ is far more altered than the structure of $\mathrm{Al}_{15}^{+}$from its neutral precursor. The difference in the ground-state geometries of anionic and neutral clusters leads to a broadening of the photo-detachment spectra and will be discussed in the later part of this section.

\section{B. Binding energy and relative stability}

The relative stability of clusters can be studied through the measurement of the mass ion intensities and fragmentation channels. However, interpretation of these results is not always unambiguous. Note that in mass spectroscopy experiments, the clusters have to be ionized before their intensities can be measured. It is not entirely clear if the measured intensity distribution of the clusters represents the stability of the charged clusters or their neutral precursors. For example, if the mass spectra are collected after the ionized clusters have time to relax to their stable configuration, the corresponding mass-ion intensities could be different from the neutral mass distribution. In addition, in order to relate the peaks in the mass spectral ion intensities to the stability of their neutral precursors, one has to assume that the charged clusters are formed by single photon ionization and that there are no photo-induced fragmentation of the clusters in the process. These difficulties are manifested in experimental observations that differ from one group to another. For example, mass spectra of $\mathrm{Al}_{n}^{+}(n=1-33)$ and $\mathrm{Al}_{n}^{-} \quad(n=5$
-37) have been measured by Leuchtner et al. ${ }^{23}$ While the authors observed enhanced peak intensity for $\mathrm{Al}_{13}^{-}$, no anomalous break in the intensity distribution was observed at $\mathrm{Al}_{3}^{+}$or $\mathrm{Al}_{7}^{+}$. On the contrary, Jarrold et al. ${ }^{20}$ found the intensity of the peak corresponding to $\mathrm{Al}_{7}^{+}$to be larger than its neighbors. Considering aluminum to be trivalent, $\mathrm{Al}_{7}^{+}$and $\mathrm{Al}_{13}^{-}$would contain 20 and 40 valence electrons, respectively. According to the jellium model these would lead to electronic shell closure and, therefore, to enhanced stability. Thus, the discrepancy regarding the anomalous peak intensity of $\mathrm{Al}_{7}^{+}$reveals that the experimental conditions are important in identifying magic numbers. Similar discrepancies also exist for $\mathrm{Al}_{14}^{+}$. While large breaks in the intensity distribution at $\mathrm{Al}_{14}^{+}$was seen in many sputtered aluminum spectra, ${ }^{39}$ neutral aluminum mass spectra, ${ }^{19,40}$ and cation spectra, ${ }^{19}$ Leuchtner et al. ${ }^{23}$ failed to observe this in their experiment. The enhanced stability of $\mathrm{Al}_{14}^{+}$is hard to understand within the jellium model since it contains 41 valence electrons and for shell closure one needs only 40 electrons. $\mathrm{Al}_{13}^{+}$, on the other hand, has 38 valence electrons and does not correspond to shell closings either. However, the geometry of $\mathrm{Al}_{13}^{+}$is more compact than the geometry of $\mathrm{Al}_{14}^{+}$(see Fig. 3) and on this ground alone, $\mathrm{Al}_{13}^{+}$would have been more stable than $\mathrm{Al}_{14}^{+}$. We will discuss the origin of this anomalous behavior later in Sec. III H of the paper. We should also point out that neither of the above two experimental groups have observed any anomalous intensity break corresponding to $\mathrm{Al}_{3}^{+}$. However, photodestruction experiments, as we shall show later, indicate a magic behavior of the $\mathrm{Al}_{3}^{+}$cluster.

An unambiguous insight into the relative stability of clusters can be gained by analyzing their energetics. We first calculate the binding energy/atom of the neutral clusters. This is defined by

$$
E_{b}=-\left[E_{n} / n-E_{0}\right],
$$

where $E_{n}$ is the total energy of the neutral cluster (given in Table II) containing $n$-atoms and $E_{0}$ is the energy of the atom. The binding energy/atom $E_{b}$ is plotted as a function of 
cluster size in Fig. 4(c). We note that the binding energy rises monotonically with cluster size and contains only a minor bump at $n=7$. The binding energy/atom for the 15atom cluster is significantly smaller than the bulk cohesive energy of $3.39 \mathrm{eV}$. Thus, the coordination number and binding energy evolve very slowly with size.

To study the relative stability, it is more instructive to analyze the first derivative of the total energy, i.e., the energy gain in adding an atom to an existing cluster. We first investigate this energy gain, hereafter referred to as the stabilization energy, for neutral clusters, namely

$$
\Delta E_{n}^{0}=-\left[\left(E_{n}^{0}-E_{n-1}^{0}\right)-E_{0}\right] .
$$

These are plotted in Fig. 5(b). The size-variation of $\Delta E_{n}^{0}$ is very different from that in alkali metal clusters. For example, there are no odd-even alternations nor any anomalous peaks corresponding to magic numbers given by the jellium model. On the contrary, $\Delta E_{n}^{0}$ rises monotonically from $n=2$ to 5 and from $n=8$ to 12 . There is a conspicuous peak at $n=7$ and $\mathrm{Al}_{14}$ is less preferred than $\mathrm{Al}_{13}$ on the ground of ener-
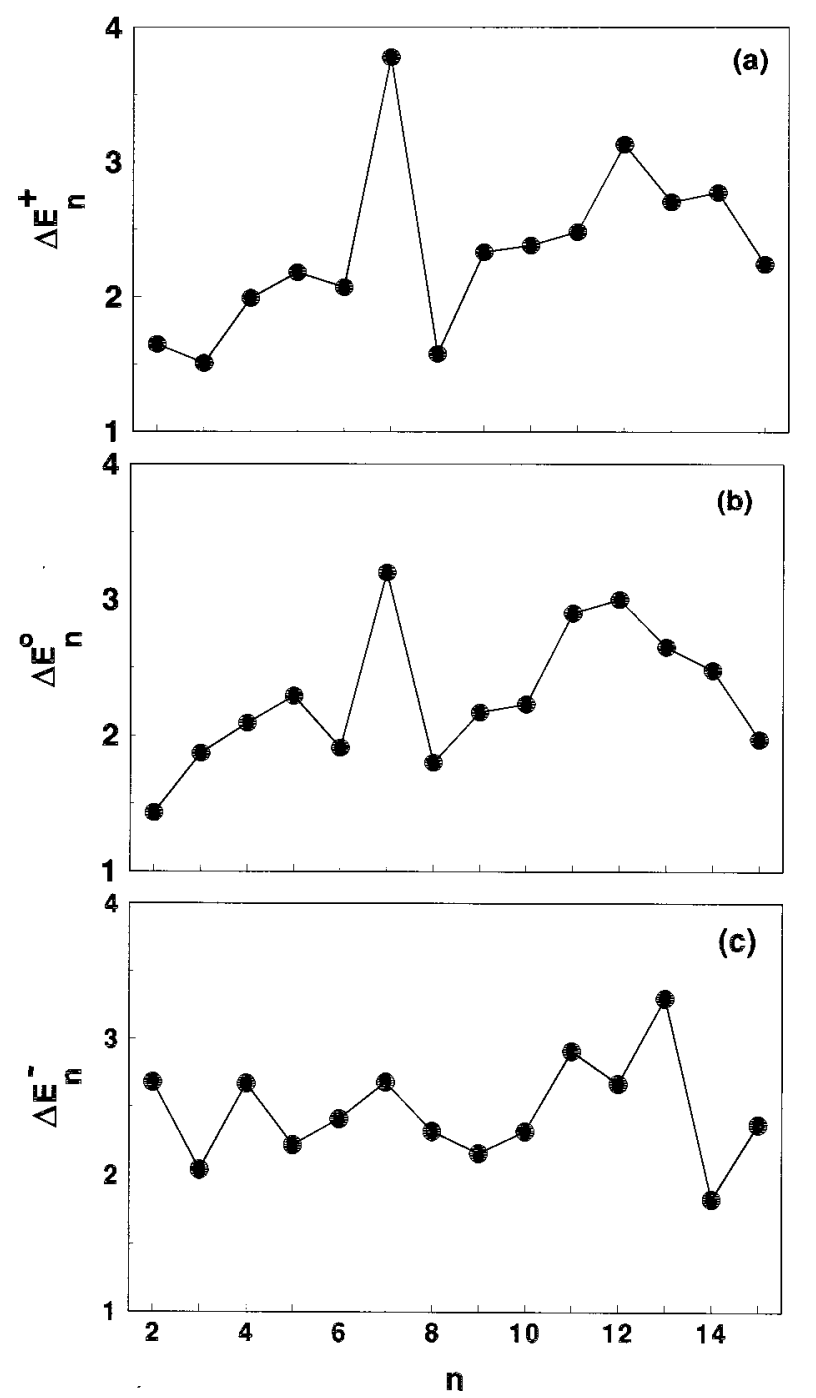

FIG. 5. Energy gain in adding an atom to a (a) positively charged cluster, $\Delta E_{n}^{+}$, (b) neutral cluster, $\Delta E_{n}^{0}$, and (c) negatively charged cluster, $\Delta E_{n}^{-}$, for $2 \leqslant n \leqslant 15$. getics. Thus, the results in Fig. 5(b) clearly indicate that the peaks in the mass ion intensities observed experimentally do not reflect the relative stability of the neutral clusters with the exception of $\mathrm{Al}_{7}$ that is controversial as discussed above.

We now study the relative stability of the positively charged clusters. In Fig. 5(a) we plot the stabilization energy as an atom is added to an existing positively charged cluster, namely

$$
\Delta E_{n}^{+}=-\left[\left(E_{n}^{+}-E_{n-1}^{+}\right)-E_{0}\right],
$$

where $E_{n}^{+}$corresponds to the total energy of the positively charged $n$-atom cluster in its ground state. These energies are given in Table II. We note that the size dependence of $\Delta E_{n}^{+}$ is similar to that seen in Fig. 5(b) except that the peak corresponding to $\mathrm{Al}_{7}^{+}$is much more pronounced. This is consistent with experimental mass ion intensities due to Jarrold et al. ${ }^{20}$ We also see that $\Delta E_{n}^{+}$for $\mathrm{Al}_{14}^{+}$is slightly larger than that for $\mathrm{Al}_{13}^{+}$while the reverse is the case for the neutral cluster. This indicates that if the mass ion intensities are collected from relaxed $\mathrm{Al}_{n}^{+}$clusters, $\mathrm{Al}_{14}^{+}$may be slightly more preferable than $\mathrm{Al}_{13}^{+}$as seen experimentally by Jarrold et al. ${ }^{20}$ This slightly enhanced stability of $\mathrm{Al}_{14}^{+}$over $\mathrm{Al}_{13}^{+}$is also consistent with the relative variations in their coordination numbers: 5.9 for $\mathrm{Al}_{14}^{+}$and 5.7 for $\mathrm{Al}_{13}^{+}$. However, we will demonstrate later that the likely reason for the enhanced mass ion intensity of $\mathrm{Al}_{14}^{+}$over that of $\mathrm{Al}_{13}^{+}$is not just because it is slightly more stable, but because $\mathrm{Al}_{13}$ has two isomers with very different ionization potentials. The absence of a peak at $\mathrm{Al}_{3}^{+}$in Fig. 5(a) indicates that the nature of bonding in this case is characteristically different from the charged alkali metal trimers as well as from larger aluminum clusters. $\mathrm{Al}_{3}^{+}$, thus, cannot be described by the jellium model.

In Fig. 5(c) we plot the stabilization energy in adding an atom to a negative ion cluster, namely

$$
\Delta E_{n}^{-}=-\left[\left(E_{n}^{-}-E_{n-1}^{-}\right)-E_{0}\right] .
$$

Here the conspicuous peak is clearly at $\mathrm{Al}_{13}^{-}$. As described earlier, $\mathrm{Al}_{13}^{-}$would have 40 valence electrons and electronic shell closure would render it enhanced stability. A closer examination reveals that $\mathrm{Al}_{7}^{-}$and $\mathrm{Al}_{11}^{-}$clusters are also special. In both the cases, $\Delta E_{n}^{-}$is larger than their neighboring clusters. For $\mathrm{Al}_{11}^{-}$, the 34 valence electrons would lead to $1 f^{14}$ shell closure and its enhanced stability in terms of jellium shell closing is understandable. But the peak at $\mathrm{Al}_{7}^{-}$is a bit puzzling. We explained the $\mathrm{Al}_{7}^{+}$peak by noting that it has 20 electrons-enough for electronic shell closure of the $2 s^{2}$ shell. In this vein, $\mathrm{Al}_{7}^{-}$would have 22 electrons and thus would not correspond to shell closure. However, if we consider aluminum to be monovalent, $\mathrm{Al}_{7}^{-}$would have 8 -electrons and then it could satisfy the electronic shell closure requirement and thus exhibit enhanced stability. Thus, one could imagine that $\mathrm{Al}_{7}$ behaves as a mixed valence system whose valence of aluminum could resonate between 1 and 3. Such behavior has been seen in rare-earth systems. ${ }^{41}$ While no clear magic peak of $\mathrm{Al}_{7}^{-}$was seen in the mass ion intensity distribution, its relative inertness towards oxygen provides some indication of its electronic shell closure. 
Leuchtner et $a l .{ }^{23}$ have found that the $\mathrm{Al}_{7}^{-}$intensity, like that of $\mathrm{Al}_{13}^{-}$, increased following its etching reaction with oxygen.

\section{Fragmentation channels}

The relative stability of clusters, especially the magic number clusters, can be studied in collision- or photoinduced fragmentation experiments. In the fragmentation process the products often carry the signature of the most stable clusters. It was predicted ${ }^{42}$ on the ground of energetics that if alkali metal clusters are fragmented, the dominant channel would always involve a magic number. For example, the dominant channels in the fragmentation of $\mathrm{Na}_{n}^{+}$ would include clusters containing $3,9,21, \ldots$ atoms. This is indeed what was observed experimentally. ${ }^{43}$ Thus, in an actual experiment if charged clusters are fragmented before they have a chance to relax to their ground states, the dominant channels may include products that are different from those resulting from the ground state of the charged clusters. These experiments have been carried out by collision induced dissociation (CID) and by photo destruction. Jarrold et al. ${ }^{20}$ have studied the fragmentation of $\mathrm{Al}_{n}^{+}(\mathrm{n}=3-26)$ by colliding the mass selected ions with argon at a center-ofmass collision energy of $5.25 \mathrm{eV}$. The authors observed that for $n \leqslant 14$, the dominant fragmentation channel included the ejection of $\mathrm{Al}^{+}$while for $n \geqslant 15$ it included the ejection of $\mathrm{Al}_{n-1}^{+}$. The only exception was for $\mathrm{Al}_{3}^{+}$which preferentially fragmented to yield $\mathrm{Al}_{2}^{+}$and $\mathrm{Al}$. For $\mathrm{Al}_{6}^{+}$, although the major fragmentation products included $\mathrm{Al}^{+}$and $\mathrm{Al}_{7}$, there was significant branching to $\mathrm{Al}$ and $\mathrm{Al}_{7}^{+}$. Similar results were also obtained by Hanley et $a .^{21}$ who performed CID experiments. These results, however, are somewhat different from those obtained by Saunders et al. ${ }^{22}$ who performed photo destruction experiments as a function of photon energies for $\mathrm{Al}_{n}^{+}$and $\mathrm{Al}_{n}^{-}(n=2-8)$. These authors found that $\mathrm{Al}_{3}^{+}$preferentially fragments to $\mathrm{Al}_{2}^{+}$and $\mathrm{Al}$ as observed by earlier authors. However, they found the predominant fragment of $\mathrm{Al}_{n}^{+}(n=4-8)$ to be $\mathrm{Al}_{n-1}^{+}$.

A theoretical understanding of the fragmentation process is difficult as there are three possible cases to consider: Spontaneous fragmentation, fragmentation after the cluster comes to thermal equilibrium with the environment, and fragmentation of the hot but vibrationaly equipartitioned cluster. Two quantities can influence the preferred fragmentation channels: The energy needed to dissociate a cluster into binary fragments and the barrier height for such a dissociation to occur. Here we examine the former, namely, the energy needed to dissociate a neutral $n$-atom cluster to $m$ and ( $n$ $-m$ ) atom clusters. This is given by

$$
\Delta E_{n m}^{0}=E_{m}^{0}+E_{n-m}^{0}-E_{n}^{0} .
$$

A similar analysis can be done for positively charged clusters by calculating the energy needed to dissociate an $n^{+}$-cluster to $m^{0}$ (neutral) and $(n-m)^{+}$(charged) fragments, namely

$$
\Delta E_{n m}^{+}=E_{m}^{0}+E_{n-m}^{+}-E_{n}^{+},
$$

where $1 \leqslant m \leqslant n-1$. However, there are two ways of how Eq. (8) can be analyzed. If the charged clusters fragment

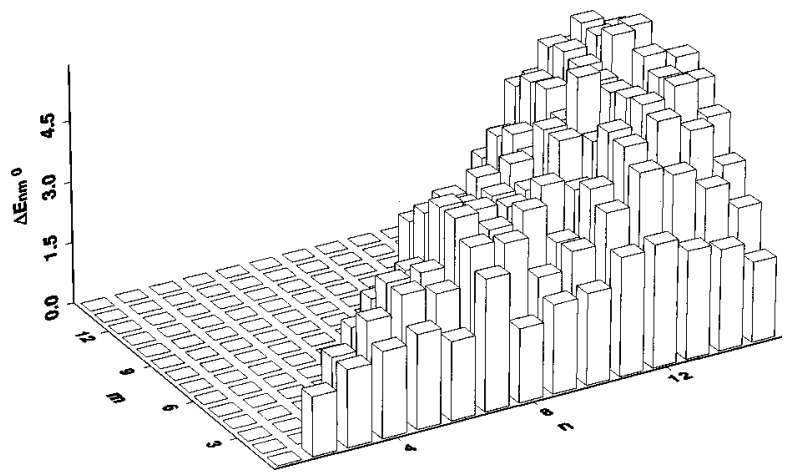

FIG. 6. Fragmentation energies of neutral clusters for various channels: $n$ $\rightarrow m,(n-m)$.

immediately following their ionization, they would not have time to relax to their ground state and $E_{n}^{+}$energies would correspond to the total energies of positively charged $n$-atom clusters having the geometry of their neutral precursors. However, if the clusters have enough time to relax before fragmentation, the total energies $E_{n}^{+}$should correspond to the ground-state (relaxed) configuration of the charged clusters. We have calculated the total energies of $\mathrm{Al}_{n}^{+}$corresponding to the unrelaxed (neutral) and relaxed (groundstate) geometries. In Table I only the energies of the ground state of $\mathrm{Al}_{n}^{+}$clusters are given.

Using the total energies of $E_{n}^{0}$ in Table I we plot $\Delta E_{n m}^{0}$ for the neutral configuration in Fig. 6. We identify the preferred channel to be the one for which $\Delta E_{n m}^{0}$ is minimum assuming that minimum $\Delta E_{n m}^{0}$ goes with minimal barrier height. The energetically most favorable channels obtained from Fig. 6 are summarized in Table III. We note that for the fragmentation of the neutral cluster, evaporation of an atom is the most dominant channel. The energetically next favorable channel is the evaporation of a dimer, but these energy costs are 0.4 to $1.8 \mathrm{eV}$ above the lowest fragmentation channel for all the clusters studied.

The situation is very different for the fragmentation of positively charged clusters. Here we have two sets of $\Delta E_{n m}^{+}$

TABLE III. Preferred fragmentation channels for neutral and charged clusters.

\begin{tabular}{ccccc}
\hline \hline \multirow{2}{*}{$\begin{array}{c}\text { Cluster } \\
\text { size } n\end{array}$} & & \multicolumn{3}{c}{$\mathrm{Al}_{n}^{+}$} \\
\cline { 3 - 4 } & $\mathrm{Al}_{n}$ & Unrelaxed & Relaxed & $\mathrm{Al}_{n}^{-}$ \\
\hline 2 & $(1,1)$ & $\left(1,1^{+}\right)$ & $\left(1,1^{+}\right)$ & $\left(1^{-}, 1\right)$ \\
3 & $(2,1)$ & $\left(1,2^{+}\right)$ & $\left(1,2^{+}\right)$ & $\left(2^{-}, 1\right)$ \\
4 & $(3,1)$ & $\left(3,1^{+}\right)$ & $\left(3,1^{+}\right)$ & $\left(3^{-}, 1\right)$ \\
5 & $(4,1)$ & $\left(4,1^{+}\right)$ & $\left(4,1^{+}\right)$ & $\left(4^{-}, 1\right)$ \\
6 & $(5,1)$ & $\left(5,1^{+}\right)$ & $\left(5,1^{+}\right)$ & $\left(5^{-}, 1\right)$ \\
7 & $(6,1)$ & $\left(6,1^{+}\right)$ & $\left(6,1^{+}\right)$ & $\left(6^{-}, 1\right)$ \\
8 & $(7,1)$ & $\left(1,7^{+}\right)$ & $\left(1,7^{+}\right)$ & $\left(7^{-}, 1\right)$ \\
9 & $(8,1)$ & $\left(8,1^{+}\right)$ & $\left(1,8^{+}\right)$ & $\left(8^{-}, 1\right)$ \\
10 & $(9,1)$ & $\left(1,9^{+}\right)$ & $\left(1,9^{+}\right)$ & $\left(9^{-}, 1\right)$ \\
11 & $(10,1)$ & $\left(1,10^{+}\right)$ & $\left(1,10^{+}\right)$ & $\left(10^{-}, 1\right)$ \\
12 & $(11,1)$ & $\left(1,11^{+}\right)$ & $\left(1,11^{+}\right)$ & $\left(11^{-}, 1\right)$ \\
13 & $(12,1)$ & $\left(12,1^{+}\right)$ & $\left(1,12^{+}\right)$ & $\left(12^{-}, 1\right)$ \\
14 & $(13,1)$ & $\left(13,1^{+}\right)$ & $\left(1,13^{+}\right)$ & $\left(13^{-}, 1\right)$ \\
15 & $(14,1)$ & $\left(1,14^{+}\right)$ & $\left(1,14^{+}\right)$ & $\left(14^{-}, 1\right)$ \\
\hline \hline
\end{tabular}


values, one corresponding to $E_{n}^{+}$for the relaxed cationic clusters and the other corresponding to $E_{n}^{+}$for the unrelaxed cationic clusters (with geometries of neutral clusters). Using Eq. (8), we have analyzed the fragmentation energies for all possible channels similar to that given in Fig. 6. For brevity, we summarize the most favored channel (i.e., the channel with minimum $\Delta E_{n m}^{+}$) in Table III. Whether the parent cluster is relaxed or not, there are two competing channels where the fragmentation product contains either an $\mathrm{Al}^{+}$or $\mathrm{Al}_{n-1}^{+}$. The energy differences between these two competing channels, however, are small and lie between 0.01 and $0.47 \mathrm{eV}$. $\mathrm{Al}_{3}^{+}$is found to preferentially fragment to $\mathrm{Al}_{2}^{+}$and $\mathrm{Al}$ irrespective of whether $\mathrm{Al}_{3}^{+}$is in its ground state or not. This agrees with the result obtained by all the experimental groups. For $n$ up to 7, the dominant product contains $\mathrm{Al}^{+}$ and this agrees with the results from collision induced experiment. ${ }^{20}$ We see no ejection of $\mathrm{Al}_{3}^{+}$as claimed by Saunders et al. ${ }^{22}$ This implies that $\mathrm{Al}_{3}^{+}$is not a magic cluster and the bonding here is not mediated by metallic nature of electrons. Note that neither the energetics in Figs. 5(a) or 5(b) nor the measured mass ion intensity gives any indication of $\mathrm{Al}_{3}^{+}$being magic. However, in contradiction with CID experiments ${ }^{20}$ and in agreement with the photo destruction experiment, $\mathrm{Al}_{8}^{+}$is found to preferentially yield $\mathrm{Al}_{7}^{+}$. As we have seen in Fig. 5(a), $\mathrm{Al}_{7}^{+}$is distinctly a magic number cluster. This has also been seen to be the case in the mass spectra. Thus, $\mathrm{Al}_{7}^{+}$should be a preferred product in the fragmentation of $\mathrm{Al}_{8}^{+}$. We note that the channels between $\mathrm{Al}^{+}+\mathrm{Al}_{7}$ and $\mathrm{Al}+\mathrm{Al}_{7}^{+}$differ by $0.07 \mathrm{eV}(0.39 \mathrm{eV})$ if the parent clusters are unrelaxed (relaxed). For clusters with $n$ $\geqslant 9$, the preferred product is $\mathrm{Al}_{n-1}^{+}$if the parent clusters are assumed to fragment from their ground state. However, if they fragment from their unrelaxed configuration, $\mathrm{Al}_{9}^{+}$, $\mathrm{Al}_{12}^{+}$, and $\mathrm{Al}_{13}^{+}$do eject an $\mathrm{Al}^{+}$ion. These discussions clearly reveal that if the charge of the fragmentation products can be ignored, the dominant channel is the ejection of an atom. Whether the atom or the daughter cluster carries the charge following fragmentation depends on the cluster size and on its geometry. The latter depends on whether fragmentation occurs from the unrelaxed or relaxed configuration of the cation. Since these two channels are energetically close, experimental conditions such as temperature and energy distribution could play an important role. This explains why different experimental groups find different fragmentation products.

Fragmentation of negatively charged clusters is even more complicated to understand than that of the positively charged clusters, since electron detachment can compete with fragmentation. This is particularly the case when the energy for electron detachment (i.e., the electron affinity) is comparable to the monomer binding energy. Saunders et l. $^{22}$ have studied the photo-fragmentation of anionic clusters $(n=2-8)$ over a range of photon energies. They have found that the neutral atom evaporation is the most dominant channel. We have calculated the energy needed to fragment $n$-atom cluster into $m^{0}$ and $(n-m)^{-}$clusters using the equation

$$
\Delta E_{n m}^{-}=E_{m}^{0}+E_{n-m}^{-}-E_{n}^{-} .
$$

TABLE IV. Calculated fragmentation energy of $\mathrm{Al}_{n}^{-}$corresponding to the most preferred pathway (ejection of a neutral atom) and the adiabatic electron detachment energy. Experimental results from two different groups are also given.

\begin{tabular}{rcccc}
\hline \hline & & \multicolumn{3}{c}{ Adiabatic detachment energy (eV) } \\
\cline { 3 - 5 }$n$ & $\begin{array}{c}\text { Fragmentation } \\
\text { energy (eV) }\end{array}$ & Theory & $\begin{array}{c}\text { Expt. 1 } \\
\text { (Ref. 28) }\end{array}$ & $\begin{array}{c}\text { Expt. 2 } \\
\text { (Ref. 30) }\end{array}$ \\
\hline 1 & & 0.13 & 0.27 & $0.44 \pm 0.01$ \\
2 & 2.68 & 1.38 & & $1.46 \pm 0.06$ \\
3 & 2.04 & 1.55 & 1.53 & $1.89 \pm 0.04$ \\
4 & 2.67 & 2.13 & 1.74 & $2.20 \pm 0.05$ \\
5 & 2.22 & 2.06 & 1.82 & $2.25 \pm 0.05$ \\
6 & 2.41 & 2.56 & 2.09 & $2.63 \pm 0.06$ \\
7 & 2.68 & 2.04 & 1.96 & $2.43 \pm 0.06$ \\
8 & 2.32 & 2.56 & 2.22 & $2.35 \pm 0.08$ \\
9 & 2.16 & 2.54 & 2.47 & $2.85 \pm 0.08$ \\
10 & 2.32 & 2.64 & 2.47 & $2.70 \pm 0.07$ \\
11 & 2.91 & 2.64 & 2.53 & $2.87 \pm 0.06$ \\
12 & 2.67 & 2.31 & 2.53 & $2.75 \pm 0.07$ \\
13 & 3.30 & 3.38 & 2.86 & $3.62 \pm 0.06$ \\
14 & 1.83 & 2.30 & 2.47 & $2.60 \pm 0.08$ \\
15 & 2.37 & 2.70 & 2.53 & $2.90 \pm 0.08$ \\
\hline \hline
\end{tabular}

The total energies for the relaxed geometries of the negatively charged clusters are given in Table II. We have analyzed fragmentation energies for all possible channels similar to that described in Fig. 6. Again, for brevity, the most favorable fragmentation channels (i.e., when $\Delta E_{n m}^{-}$is minimum) are summarized in Table III. We note that among all the fragmentation channels, ejection of a neutral atom is the most preferred pathway-in agreement with experiment. The next energetically preferred channel is the emission of a neutral dimer, but these energies are 0.4 to $1.5 \mathrm{eV}$ higher than the most preferred pathway.

Saunders $e t a l^{22}$ also observed that for $\mathrm{Al}_{3}^{-}$, very little (less than 1\%) fragment signal was detected for all photon energies suggesting that electron detachment rather than fragmentation is the dominant photo destruction mechanism. This is consistent with our results. We will show in the following that the electron affinity of $\mathrm{Al}_{3}^{-}$is $1.55 \mathrm{eV}$ (i.e., it will cost $1.55 \mathrm{eV}$ for $\mathrm{Al}_{3}^{-}$to detach its electron) while $\Delta E_{n m}^{-}$for fragmentation into neutral $\mathrm{Al}$ atom is $2.04 \mathrm{eV}$. We find similarly that the electron detachment is the most dominant photo destruction mechanism for $\mathrm{Al}_{n}^{-}(n=4,5,7,11,12,13)$. However, the energy differences between electron detachment and fragmentation lie in the range of $0.16-0.54 \mathrm{eV}$ (see Table IV) suggesting that these two processes compete during the photo destruction experiment. This is, indeed, what has been observed experimentally by Saunders et al. who found that at low photon energies fragmentation is strong and it generally decreases with photon energies.

\section{Electronic structure}

The evolution of the electronic structure can be probed by calculating the angular characteristics of the highest molecular orbital (HOMO) as well as the energy gap between HOMO and the lowest unoccupied molecular orbital (LUMO). To gain insight into the evolution of the $s$ - $p$ overlap, we have analyzed the $s$ and $p$ content of the highest 


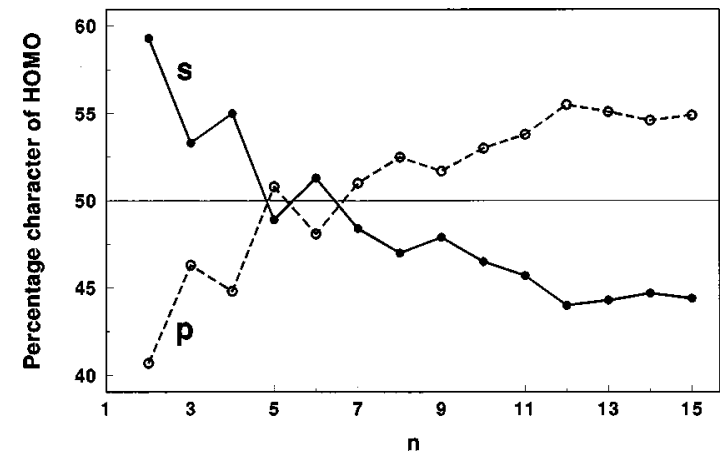

FIG. 7. Concentration of $s$ and $p$-type electrons in the highest occupied molecular orbital (HOMO) of aluminum clusters.

occupied molecular orbital (HOMO). The results are plotted in Fig. 7. Note that in the atom, the valence electrons are composed of $66 \% s$-electrons and $33 \% p$-electrons $\left(3 s^{2} 3 p^{1}\right)$. We see that with increasing cluster size the $s$ content begins to decrease with a corresponding increase in the $p$ content. For clusters with less than 5 atoms, the HOMO is clearly $s$-like while for clusters containing more than 7
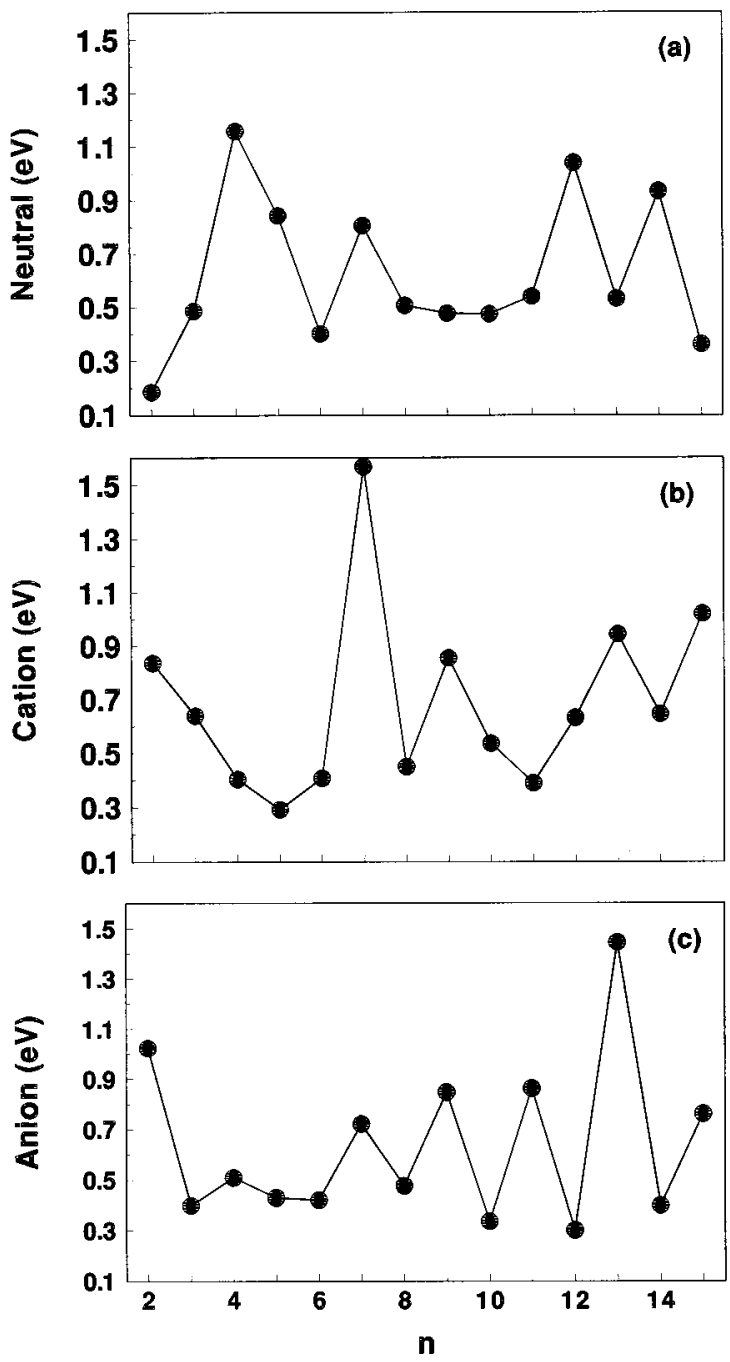

FIG. 8. HOMO-LUMO gap of (a) neutral, (b) positively charged, and (c) negatively charged clusters. atoms it is $p$-like. Clusters with $5 \leqslant n \leqslant 7$ represent a region of transition. It is in this size range that the geometry becomes three dimensional and is accompanied by a sudden increase in the number of bonds and coordination number. Thus, if we consider aluminum to be trivalent in a cluster size where the $s$ and $p$ states overlap, this would make the critical size to be $n=7$. Note that this is consistent with our interpretation of the mass-ion intensity and fragmentation. We will also show in the following that the photo detachment spectra ${ }^{30}$ of $\mathrm{Al}_{7}^{-}$is consistent with our observation of hybridized $s$ and $p$ states at this size.

To understand if this electronic structure is manifested in the HOMO-LUMO gap, we have calculated the energy gaps for all the neutral, cationic, and anionic clusters. The results are presented in Figs. 8(a)-8(c). Note that a cluster with a closed electronic shell is characterized not only by a large HOMO-LUMO gap but also by a filled HOMO. It also exhibits enhanced binding energy compared to their neighbors. We see from Fig. 8(a) that the HOMO-LUMO gaps in neutral clusters are particularly large for $\mathrm{Al}_{4}, \mathrm{Al}_{7}, \mathrm{Al}_{12}$, and $\mathrm{Al}_{14}$. None of these clusters contain the number of valence electrons necessary to form closed electronic shells in a jellium model as described earlier. We see from Fig. 5(b) that although the energy gain, $\Delta E_{n}^{0}$ shows a peak for $\mathrm{Al}_{7}$ and $\mathrm{Al}_{12}$, it has no characteristic feature for $\mathrm{Al}_{4}$ and $\mathrm{Al}_{14}$.

The situation is somewhat different for charged clusters. For positively charged $\mathrm{Al}$ clusters [see Fig. 8(b)] conspicuous peaks in the HOMO-LUMO gaps do exist for $\mathrm{Al}_{7}^{+}$, $\mathrm{Al}_{9}^{+}, \mathrm{Al}_{13}^{+}$, and $\mathrm{Al}_{15}^{+}$. The peak in $\mathrm{Al}_{7}^{+}$is easily understood in the jellium model as the cluster would contain 20 valence electrons, just enough for electronic shell closure. We see from Fig. 5(a) that $\Delta E_{n}^{+}$for $\mathrm{Al}_{7}^{+}$is large and thus the large HOMO-LUMO gap is consistent with its enhanced stability. No such correlation exists for $\mathrm{Al}_{9}^{+}, \mathrm{Al}_{13}^{+}$, and $\mathrm{Al}_{15}^{+}$.

For negatively charged clusters, the HOMO-LUMO gap exhibit odd-even alteration for $n \geqslant 6$ with odd-atom clusters having larger HOMO-LUMO gaps than even-atom clusters [see Fig. 8(c)]. Of particular interest are the peaks at $\mathrm{Al}_{13}^{-}$, $\mathrm{Al}_{7}^{-}$, and $\mathrm{Al}_{11}^{-}$. It is clear that $\mathrm{Al}_{13}^{-}$with 40 electrons can fill the electronic shells and hence has a very large HOMOLUMO gap. If aluminum in $\mathrm{Al}_{7}$ can also be regarded as mono-valent as discussed earlier, $\mathrm{Al}_{7}^{-}$would have 8 valence electrons and hence its large HOMO-LUMO gap would be consistent with electronic shell closures. Similarly $\mathrm{Al}_{11}^{-}$with 34 electrons also is a cluster with closed electronic shell and hence should have a large HOMO-LUMO gap and it does. Note that these are consistent with the relative stabilities of these clusters as seen from the systematics in $\Delta E_{n}^{-}$in Fig. 5(c). However, the large HOMO-LUMO gap in $\mathrm{Al}_{9}^{-}$, is neither consistent with the electronic shell closure nor with their relative stabilities. $\mathrm{Al}_{15}^{-}$, on the other hand, shows large HOMO-LUMO gap which is consistent with its greater stability than $\mathrm{Al}_{14}^{-}$, but it does not correspond to electronic shell closure.

\section{E. Magnetic properties}

The magnetic properties of aluminum clusters were first studied by Cox et al. ${ }^{19}$ by measuring the deflection of these 
clusters in a Stern-Gerlach magnetic field. Because aluminum is an $s-p$ electron metal, one would not expect these clusters to be magnetic. However, odd-atom clusters of $\mathrm{Al}$ would at least have one unpaired spin and thus, would possess $1 \mu_{B}$ magnetic moment at the least. This corresponds to a spin multiplicity of $M=2 S+1=2$. Consequently, they would undergo deflection in a Stern-Gerlach field. For evenatom clusters, the even-number of electrons would either give rise to $0 \mu_{B}$ magnetic moment (spin multiplicity of 1 ) or higher. Cox et al. ${ }^{19}$ found that the spin multiplicities of small Al clusters $(n \leqslant 10)$ were 2 for odd-atom clusters and 3 for even-atom clusters. The authors were unable to determine the spin multiplicity of larger $(n>10)$ clusters as their experiment was limited by the finite deflecting power of the magnet and the spatial extent of the ionizing laser and the cluster beam.

We calculated the total energies corresponding to optimized structures of neutral $\mathrm{Al}$ clusters for spin-multiplicities of 2 and 4 for odd-atom clusters and 1 and 3 for even-atom clusters. The energetically preferred spin multiplicities are given in Table II. Note that our results are in complete agreement with the experimental data of Cox et al. Furthermore, preferred spin multiplicities of $\mathrm{Al}_{12}$ and $\mathrm{Al}_{14}$ are 1 and thus the clusters are nonmagnetic. Consequently, they would not deflect in a Stern-Gerlach field no matter what improvements are made in the experimental set up. Note that these experiments cannot be performed for charged clusters. It is worth noting that a recent local spin-density calculation by Akola et al. ${ }^{15}$ found the ground state to have minimum total spin, i.e., $S=0$ for even- and $S=\frac{1}{2}$ for odd-atom clusters in the range of 3-23 atoms. This result is inconsistent with experiment as well as our results for even-atom clusters with $n \leqslant 10$.

\section{F. Ionization potential}

The ionization potential measures the energy difference between the ground state of the neutral and the ionized clusters. If the ionized cluster has the same geometry as the ground state of the neutral, the ionization energy corresponds to the vertical ionization potential. On the other hand, the energy difference between the ground state of the cation and ground state of the neutral is referred to as the adiabatic ionization potential. Thus, the vertical ionization potential is always larger than the adiabatic ionization potential and the energy difference between them is an indication of the energy gain due to structural relaxation. Note that in the jellium model where the geometry is ignored, these two numbers are the same. Since experimentally one normally measures the vertical ionization potential, we discuss only this aspect, although the adiabatic ionization potential can be easily computed from the total energies given in Table II. It is sufficient to mention that the energy gain by relaxation of the geometry of the ionized cluster lies in the range of $0.04-0.47 \mathrm{eV}$ (0.004-0.030 eV/atom) for the clusters studied. The vertical ionization potentials were calculated by evaluating the total energy of the ionized cluster at the neutral geometry.

In the jellium model, the ionization potential is given by the formula

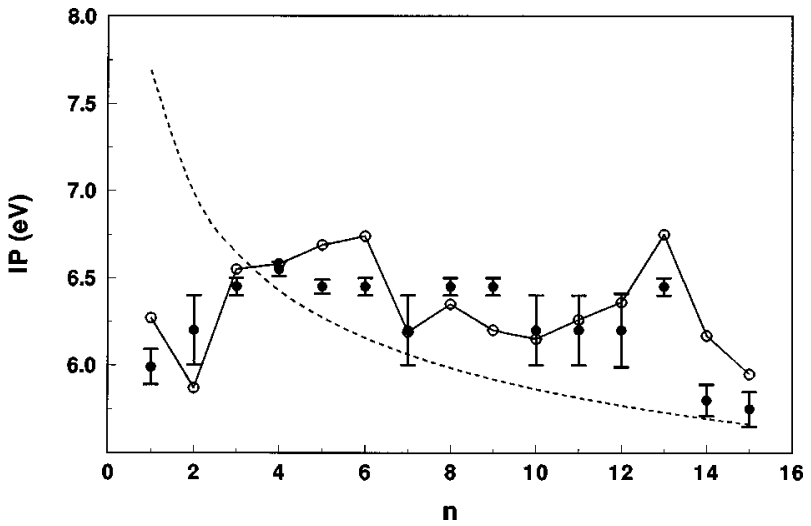

FIG. 9. Comparison of experimental (Ref. 24) ionization potentials (filled circles with error bars) with those calculated from first principles theory (open circles joined by solid line) and jellium model (dashed line).

$$
W_{R}=W_{\infty}+\frac{3 e^{2}}{8 R},
$$

where $W_{\infty}$ is the bulk work function $(4.25 \mathrm{eV})$ and $R$ is the radius of the cluster. Assuming aluminum to be trivalent, $R$ $=(3 n / 16 \pi){ }^{1 / 3} L$ where $n$ is the number of atoms and $L$ is the lattice constant (4.05 $\AA$ ). The result of Eq. (10) is compared with experimental data ${ }^{24}$ as well as those obtained from our first principles calculations in Fig. 9. We note that the jellium model does not fare very well in accounting for the sizedependence of the experimental data. ${ }^{24}$ Based on this deviation, one can conclude that the jellium model is inadequate in explaining the ionization potentials. de Heer et al. ${ }^{25}$ have measured the dipole polarizabilities of $\mathrm{Al}_{n}$ clusters $(n \leqslant 61)$ and observed that the clusters containing less than 40 atoms did not behave like jellium. However, as indicated before and as we will demonstrate below, certain characteristics are in agreement with the electron shell closing argument.

The most detailed calculations of the ionization potentials (IP) up to $n=10$ were carried out by Jones ${ }^{14}$ using the local spin-density approximation. Similar calculations have been recently performed by Akola et al. ${ }^{15}$ for $2 \leqslant n \leqslant 23$. The results are in general agreement with experiment and account for the trend that it initially increases up to $n=6$ and has a significant dip at $n=7$. The low IP of $\mathrm{Al}_{7}$ is consistent with the electronic shell structure since it has one additional electron beyond the shell closing requirement. Our calculated ionization potentials are in better agreement with experiment ${ }^{24}$ than any other previous calculation. There are two important features to be noted in Fig. 9. First, the ionization potentials of clusters up to $n=14$ are higher than that of the atom $(6.27 \mathrm{eV})$. In the alkali metal clusters, this is just the opposite- the atom has the highest ionization potential. Second, there are no odd-even alternations in the ionization potential as a function of size with the exception of a conspicuous dip at $n=7$ and a peak at $n=13$. In alkali clusters, the IP's exhibit odd-even alternation with higher IP being associated with even-number clusters. We remind the reader that the fragmentation of $\mathrm{Al}_{n}^{+}$clusters yielded $\mathrm{Al}^{+}$as the preferred product for $n \leqslant 14$. This is because the IP's of $\mathrm{Al}_{n}$ $(n \leqslant 14)$ clusters are larger than that of the $\mathrm{Al}$ atom as seen from Fig. 9. 


\section{G. Electron affinities}

We next discuss the electron detachment from negatively charged clusters. Here, a size selected negative ion cluster is crossed with a fixed frequency laser and the photodetached electron is energy analyzed. From this, one can measure the binding energy of the electron in the negative ion cluster. The resulting photo-emission spectra (PES) carries information on the electronic structure of the neutral cluster. The latest work in this series is due to Li et al ${ }^{30}$ who studied photoelectron spectroscopy of $\mathrm{Al}_{n}^{-}(n=1-162)$ at $6.42 \mathrm{eV}$ photon energy. They observed weak shell closure features at $\mathrm{Al}_{11}^{-}$and a much stronger shell closure effect at $\mathrm{Al}_{13}^{-}$. They also observed a broadening of the $\mathrm{Al} 3 s$ and $3 p$ levels with cluster size. These levels begin to overlap completely at $n=9$. Before we discuss our results, we should emphasize that what the experiment actually measures is the difference between the total energy of the ground state of the anion and the total energy of the ground state of the neutral as well as its electronically and vibrationally excited states. Very often the experimental PES spectra is used to comment on the evolution of the electron density of states of the neutral clusters. There are difficulties associated with the quantitative meaning of this interpretation. First, it relies on the validity of the Koopman's theorem for clusters. Secondly, in density-functional calculations the energy levels have no fundamental meaning. In addition, if the anions contain energetically degenerate isomers, the interpretation of the experimental data becomes further complicated. In our discussion of the electron detachment, we use the total energies of the anionic and neutral clusters.

The photo detachment spectra normally provides two different energies - the vertical and adiabatic electron affinity. The vertical electron affinity is the difference in the energy between the ground state of the anion and the energy of the neutral cluster having the anionic geometry. Transitions are allowed as long as $\Delta M= \pm 1$ where $M=2 S+1$ is the spin multiplicity. The adiabatic electron affinity, on the other hand, is the difference in the total energy between the ground state of the anion and the neutral cluster.

In Table IV we compare the calculated adiabatic electron affinities with data from two different sets of experiment ${ }^{28,30}$. We note that the agreement between theory and experiment is very good and is within the same range as it is between the two sets of experimental data. The electron affinity of $\mathrm{Al}_{13}$, in particular, is interesting. It is the highest among all the clusters studied. Note that $\mathrm{Al}_{13}$ with 39 valence electrons needs just one electron to close its outermost shell according to the jellium model. Thus, in analogy with the halogen atoms, $\mathrm{Al}_{13}$ should have a high electron affinity. Indeed it does!

To study the vertical electron affinities we have calculated the total energies of $\mathrm{Al}_{n}$ neutral clusters having the geometry of $\mathrm{Al}_{n}^{-}$, but spin multiplicities that differ by $\Delta M$ $= \pm 1$ from the ground state anion. In Table $\mathrm{V}$ we compare these energies with those obtained from the experimental photo detachment spectra for clusters containing up to 15 atoms. Note that these agree very well with the experimental
TABLE V. Vertical electron affinity $(\mathrm{eV})$ of $\mathrm{Al}_{n}^{-}$clusters corresponding to transitions from anion to neutral clusters with $\Delta M= \pm 1$.

\begin{tabular}{cccc}
\hline \hline Cluster size, $n$ & Transition & Theory & Experiment (28) \\
\hline 2 & $4 \rightarrow 3$ & 1.44 & 1.55 \\
& $4 \rightarrow 5$ & 3.10 & 3.25 \\
3 & $3 \rightarrow 2$ & 1.63 & 1.90 \\
& $3 \rightarrow 4$ & 1.66 & 2.15 \\
4 & $2 \rightarrow 3$ & 2.19 & 2.25 \\
& $2 \rightarrow 1$ & 3.42 & 3.35 \\
5 & $1 \rightarrow 2$ & 2.13 & 2.30 \\
6 & $2 \rightarrow 1$ & 2.65 & 2.70 \\
& $2 \rightarrow 3$ & 2.70 & $\cdots$ \\
7 & $1 \rightarrow 2$ & 2.36 & 2.55 \\
8 & $2 \rightarrow 1$ & 2.41 & 2.45 \\
& $2 \rightarrow 3$ & 2.88 & 2.80 \\
9 & $1 \rightarrow 2$ & 2.76 & 2.90 \\
10 & $2 \rightarrow 1$ & 2.71 & 2.85 \\
& $2 \rightarrow 3$ & 2.84 & 3.10 \\
11 & $1 \rightarrow 2$ & 2.73 & 2.90 \\
12 & $2 \rightarrow 1$ & 2.73 & 2.80 \\
& $2 \rightarrow 3$ & 3.00 & 3.15 \\
13 & $1 \rightarrow 2$ & 3.57 & 3.75 \\
14 & $2 \rightarrow 1$ & 2.49 & 2.70 \\
& $2 \rightarrow 3$ & 2.96 & 3.20 \\
15 & $1 \rightarrow 2$ & 2.84 & 3.00 \\
\hline \hline
\end{tabular}

data-considering that our calculated energies have no adjustable parameters.

\section{$\mathrm{H}$. Isomerism in the $\mathrm{Al}_{13}$ and $\mathrm{Al}_{15}$ clusters}

As discussed earlier, the intensity of the mass ion peak corresponding to $\mathrm{Al}_{13}$ was expected to be larger than that due to $\mathrm{Al}_{14}$ because of its more compact geometry. However, this is opposite to the experimental result where the mass ion intensity of $\mathrm{Al}_{14}^{+}$was found to be larger than that of $\mathrm{Al}_{13}^{+}$. As we indicated earlier, $\mathrm{Al}_{13}$ cluster has two nearly degenerate isomers: Jahn-Teller distorted icosahedron [Fig. 10(a)] and decahedron [Fig. 3(a)]. The Jahn-Teller distortion can be seen more clearly by viewing a two-dimensional projection of the structure as given in Fig. 10(b). Note that the atoms in the outer circle are not equidistant from one another. The ionization potential of the icosahedric structure is $6.75 \mathrm{eV}$ while that of the decahedron is $5.91 \mathrm{eV}$. The experimental result is in between these two values, namely $6.45 \pm 0.05 \mathrm{eV}$. Thus in a mass spectroscopy experiment that uses a fixed frequency laser to ionize the clusters with radiation energy lying between the two ionization potentials, only one of these isomers can be detected. We believe that the decahedron is more likely to populate the mass ion intensity as it has a lower ionization potential. Additional evidence for the probable existence of the decahedral structure has recently been shown ${ }^{44}$ by comparing the calculated ionization potential of $\mathrm{Al}_{13} \mathrm{Na}$ with experiment. ${ }^{45}$ Thus, the anomaly in the mass ion intensity of $\mathrm{Al}_{13}$ is not because $\mathrm{Al}_{13}$ is less abundant than $\mathrm{Al}_{14}$ but probably because a fraction of $\mathrm{Al}_{13}$ clusters have gone undetected. A calculation of the height of the potential barrier separating these two structures could give insight on the probability of the formation of these isomers. However, as these structures are Jahn-Teller distorted, such a calculation cannot be performed. 


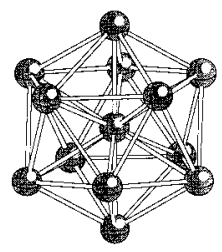

(a)

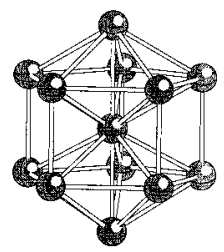

(b)

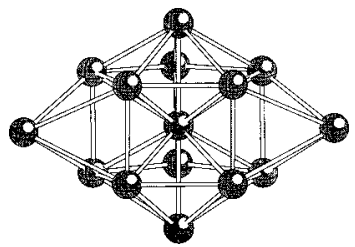

(c)

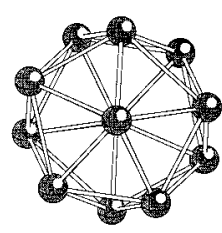

(d)

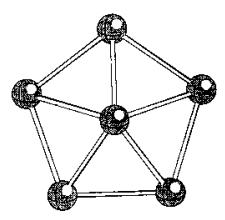

(e)

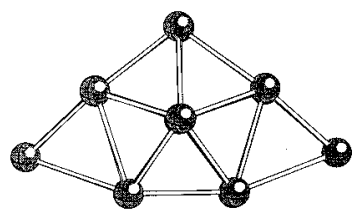

(f)
FIG. 10. Isomers of (a) $\mathrm{Al}_{13}$, (b) $\mathrm{Al}_{13}^{-}$, and (c) $\mathrm{Al}_{15}$ clusters. The projections of these clusters are given in (d)-(f), respectively to show that while the icosahedric $\mathrm{Al}_{13}$ is distorted, the other isomers are very symmetric in structure.

In a similar manner, $\mathrm{Al}_{13}^{-}$has two isomers: $\mathrm{A}$ perfect icosahedron [Fig. 3(c)] and a perfect decahedron [Fig. 10(b)]. The Jahn-Teller distortion present in the neutral cluster disappears due to the closed shell structure of the anion. This can be seen clearly in its two-dimensional projection [Fig. 10(e)]. Unlike in the case of the neutral $\mathrm{Al}_{13}$ cluster where the two isomers differed by an energy of $0.43 \mathrm{eV}$, the two isomers of the anion differ in energy by $0.12 \mathrm{eV}(0.01$ eV/atom). Clearly these two structures are degenerate. To determine the barrier height that may separate the two isomers of $\mathrm{Al}_{13}^{-}$, we calculated the total energy of the $\mathrm{Al}_{13}^{-}$ cluster as it transforms from the icosahedron to the decahedral structure. This can be done easily by rotating the top pentagon in Fig. 10(b) through an angle $\theta$ about an axis passing through the central atom and the top and bottom caps of the pentagons. At $\theta=36^{\circ}$, the decahedron transforms into an icosahedron. Since $\mathrm{Al}_{13}^{-}$remains undistorted due to its closed electronic shell structure, we simply optimized the radial distance for each value of $\theta$ used during the process of twisting. This process was repeated for steps of $\Delta \theta=6^{\circ}$ till the transformation was complete. We found the variation of the total energy with $\theta$ to be essentially flat as the energy decreased monotonically from the icosahedron to the decahedron by only $0.12 \mathrm{eV}$. This amounts to a force of 0.00016 a.u./Bohr which is much smaller than the threshold $(0.00045$ a.u./Bohr) imposed on our geometry optimization procedure. Thus the existence of $\mathrm{Al}_{13}^{-}$in two isomeric forms is not because there is an energy barrier protecting them from each other, but because the forces to drive them in either direction are vanishingly small. The adiabatic electron affinity of the icosahedric structure is $3.38 \mathrm{eV}$ while that of the decahedral structure is $2.95 \mathrm{eV}$. In this case, the result of the icosahedric structure is closer to the most recent experimental value of $3.62 \pm 0.06 \mathrm{eV}$. Signature of the isomerism of the anion in the photo detachment spectra of $\mathrm{Al}_{13}^{-}$should be critically examined.

Since recent calculations ${ }^{15}$ have found the ground state of $\mathrm{Al}_{15}$ to be a bicapped decahedron [Fig. 10(c)], we have calculated the total energy of this cluster by forcing it to assume this geometry while reoptimizing the bond lengths. We see once again that the Jahn-Teller distortions in the central decahedron disappear. This can be seen more clearly in the two-dimensional projection in Fig. 10(f) where the top and bottom pentagons coincide and the three central atoms along the (001) direction lie on top of each other. The energy of this structure is only $0.16 \mathrm{eV}$ higher than the ground state structure given in Fig. 3(a). This amounts to an energy difference of $0.01 \mathrm{eV} /$ atom and thus these two structures can be considered to be energetically degenerate within the accuracy of our calculation. We have calculated the vertical ionization potential of this isomer of $\mathrm{Al}_{15}$ to see if it shares the same behavior as that in the $\mathrm{Al}_{13}$ isomers. Interestingly, the vertical ionization potential of the isomer in Fig. 10(c) is $5.92 \mathrm{eV}$ while that of the ground-state structure [Fig. 3(a)] is $5.95 \mathrm{eV}$. This implies that both of the isomers must be present in the mass spectra.

\section{CONCLUSIONS}

Using density functional theory and generalized gradient approximation, we have calculated the ground-state geometries of neutral, positively charged, and negatively charged aluminum clusters containing up to 15 atoms. The evolution of the binding energy, atomic and electronic structure, ionization potentials, fragmentation channels, and electron affinities were calculated and compared with experiment. The results can be summarized as follows: (1) The equilibrium geometries bear no resemblance to the bulk lattice structure although the nearest-neighbor distance equals the bulk value by the time $n=15$. (2) The coordination number is significantly smaller not only from the bulk value but also from the close-packed $\langle 111\rangle$ surface. Moreover, the coordination number undergoes a significant jump when the cluster changes from two to three dimensions as well as when it develops an interior atom. (3) The binding energy/atom evolves monotonically and is far below the bulk cohesive energy even for the largest cluster studied. (4) The stabilization energy, $\Delta E$ in adding an atom to an existing cluster shows a distinct peak at $n=7$ irrespective of whether the cluster is neutral or charged. This establishes $\mathrm{Al}_{7}^{+}$as a magic number cluster and it is consistent with the electronic shell closure predicted by the jellium model. Similarly, the peaks in $\Delta E$ at $\mathrm{Al}_{11}^{-}$and $\mathrm{Al}_{13}^{-}$are consistent with shell closings. (5) The fragmentation channels are governed by the underlying electronic structure and energetics of the clusters. The neutral and negative ion clusters fragment by preferentially ejecting a neutral atom. For some of the anionic clusters, the electron detachment competes with fragmentation. For the positively charged clusters, the preferential channel also in- 
volves the emission of an atom, but whether the atom or the daughter cluster carries the positive charge depends on the size of the parent cluster. (6) The electronic structure in clusters containing less than 7 atoms does not resemble the jellium model. The $s$ and $p$ content of the highest occupied molecular orbital changes from primarily $s$-like $(66 \%$ in the free atom) to $s$ - and $p$-like (50\% $s$ and 50\% $p$ ) at $n=6,7$ to more $p$-like $(>50 \%)$ for $n \geqslant 8$. This change is accompanied by a structural transformation where the clusters become three dimensional with a concomitant increase in the coordination number. (7) The HOMO-LUMO gaps in the neutral clusters do not correlate with the electronic shell closure or with their relative stabilities. However, in charged clusters such as $\mathrm{Al}_{7}^{+}, \mathrm{Al}_{7}^{-}, \mathrm{Al}_{11}^{-}$, and $\mathrm{Al}_{13}^{-}$, the conspicuously large HOMO-LUMO gaps have strong correlation with their relative stabilities and are dictated by the electronic shell closure. (8) Odd-atom clusters carry a magnetic moment of $1 \mu_{B}$. On the other hand, even-atom clusters with $n \leqslant 10$ have a magnetic moment of $2 \mu_{B}$ while $\mathrm{Al}_{12}$ and $\mathrm{Al}_{14}$ are not magnetic. (9) The ionization potentials of $\mathrm{Al}_{n}$ clusters exhibit a strange pattern. The clusters have larger ionization potentials compared to atom for $n \leqslant 13$ and show no odd-even alternation as seen in alkali metal clusters. (10) The adiabatic electron affinity increases generally with cluster size and exhibits a conspicuous peak at $\mathrm{Al}_{13}^{-}$. The electron affinity of $\mathrm{Al}_{13}$ is very close to that of chlorine and establishes the fact that the chemistry of $\mathrm{Al}_{13}$ may be the same as that of a halogen atom. (11) The vertical electron affinities and the adiabatic ionization potentials are very close to adiabatic electron affinities and vertical ionization potentials respectively suggesting that in general, the geometries of neutral clusters do not change significantly when an electron is either attached or removed from it. (12) All the results calculated here contain no adjustable parameters. Thus, the agreement between our theoretical results with all available experimental data to date not only provides the confidence regarding the accuracy of our calculated geometries, but also demonstrates the power of theory being an independent tool.

In summary, we believe that quantitative understanding of the evolution of the properties of metal clusters containing at least a couple of dozen atoms is at hand and that the current theory has predictive capability so as to guide future experiments.

\section{ACKNOWLEDGEMENTS}

This work is partly supported by a grant from the Department of Energy (DEFG02-96ER45579). We are grateful to Professor R. Johnston for providing the coordinates of Al clusters obtained from their classical molecular-dynamics simulation. We thank Professor L. S. Wang for providing details of his experimental results.

${ }^{1}$ T. G. Dietz, M. A. Duncan, D. E. Powers, and R. E. Smalley, J. Chem. Phys. 74, 6511 (1981)

${ }^{2}$ See Physics and Chemistry of Finite Systems: From Clusters to Crystals, edited by P. Jena, S. N. Khanna, and B. K. Rao (Kluwer Academic, Dordrecht, The Netherlands, 1992).
${ }^{3}$ U. Rothlisberger and W. Andreoni, J. Chem. Phys. 94, 8129 (1991).

${ }^{4}$ W. D. Knight, K. Clemenger, W. A. de Heer, W. A. Saunders, M. Y. Chou, and M. L. Cohen, Phys. Rev. Lett. 52, 2141 (1984).

${ }^{5}$ B. K. Rao and P. Jena, Phys. Rev. B 32, 2058 (1985).

${ }^{6}$ N. D. Bhasker, R. P. Frueholz, C. M. Klimeak, and R. A. Cook, Phys. Rev. B 36, 4418 (1987).

${ }^{7}$ M. Y. Chou and M. L. Cohen, Phys. Lett. A 113, 420 (1986).

${ }^{8}$ K. Jug, H. P. Schluff, H. Kupka, and R. Iffert, J. Comput. Chem. 9, 803 (1988).

${ }^{9}$ G. Pacchioni and J. Koutecky, Ber. Bunsenges. Phys. Chem. 88, 242 (1984).

${ }^{10}$ T. H. Upton, J. Chem. Phys. 86, 7054 (1987).

${ }^{11}$ L. G. M. Petterson, C. W. Bauschlicher, Jr., and T. Halicioglu, J. Chem. Phys. 87, 2205 (1987).

${ }^{12}$ H. P. Cheng, R. S. Berry, and R. L. Whetten, Phys. Rev. B 43, 10647 (1991).

${ }^{13}$ J. Y. Yi, D. J. Oh, and J. Bernhole, Phys. Rev. Lett. 67, 1594 (1991).

${ }^{14}$ R. O. Jones, J. Chem. Phys. 99, 1194 (1993).

${ }^{15}$ J. Akola, H. Häkkinen, and M. Manninen, Phys. Rev. B 58, 3601 (1998).

${ }^{16}$ R. Ahlrichs and S. D. Elliott, Phys. Chem. Chem. Phys. 1, 13 (1999).

${ }^{17}$ S. N. Khanna and P. Jena, Phys. Rev. Lett. 69, 1664 (1992).

${ }^{18}$ X. G. Gong and V. Kumar, Phys. Rev. Lett. 70, 2078 (1993).

${ }^{19}$ D. M. Cox, D. J. Trevor, R. L. Whetten, E. A. Rohlfing, and A. Kaldor, J. Chem. Phys. 84, 4651 (1986).

${ }^{20}$ M. F. Jarrold, J. E. Bower, and J. S. Kraus, J. Chem. Phys. 86, 3876 (1987).

${ }^{21}$ L. Hanley, S. Ruatta, and S. Anderson, J. Chem. Phys. 87, 260 (1987).

${ }^{22}$ W. A. Saunders, P. Fayet, and L. Wöste, Phys. Rev. A 39, 4400 (1989).

${ }^{23}$ R. E. Leuchtner, A. C. Harms, and A. W. Castleman, Jr., J. Chem. Phys. 94, 1093 (1991); 91, 2753 (1989).

${ }^{24}$ K. E. Schriver, J. L. Persson, E. C. Honea, and R. L. Whetten, Phys. Rev. Lett. 64, 2539 (1990).

${ }^{25}$ W. A. de Heer, P. Milani, and A. Chatelain, Phys. Rev. Lett. 63, 2834 (1989).

${ }^{26}$ G. Ganteför, M. Gausa, K. H. Meiwes-Broer, and H. O. Lutz, Z. Phys. D 9, 253 (1988).

${ }^{27}$ G. Ganteför, K. H. Meiwes-Broer, and H. O. Lutz, Phys. Rev. A 37, 2716 (1988).

${ }^{28}$ K. J. Taylor, C. L. Pettiette, M. J. Graycraft, O. Chesnovsky, and R. E. Smalley, Chem. Phys. Lett. 152, 347 (1988).

${ }^{29}$ A. Nakajima, K. Hoshino, T. Naganuma, Y. Sone, and K. Kaya, J. Chem. Phys. 95, 7061 (1991).

${ }^{30}$ X. Li, H. Wu, X. B. Wang, and L. S. Wang, Phys. Rev. Lett. 81, 1090 (1998).

${ }^{31}$ P. J. Hay and W. R. Wadt, J. Chem. Phys. 82, 299 (1985).

${ }^{32}$ B. K. Rao and A. K. Ray, J. Phys.: Condens. Matter 9, 2859 (1997).

${ }^{33}$ Gaussian 94, Revision B. 1, M. J. Frisch, G. W. Trucks, H. B. Schlegel, P. M. W. Gill, B. G. Johnson, M. A. Robb, J. R. Cheeseman, T. Keith, G. A. Petersson, J. A. Montgomery, K. Raghavachari, M. A. Al-Laham, V. G. Zakrzewski, J. V. Ortiz, J. B. Foresman, J. Closlowski, B. B. Stefanov, A. Nanayakkara, M. Challacombe, C. Y. Peng, P. Y. Ayala, W. Chen, M. W. Wong, J. L. Andres, E. S. Replogle, R. Gomperts, R. L. Martin, D. J. Fox, J. S. Binkley, D. J. Defrees, J. Baker, J. P. Stewart, M. Heads-Gordon, C. Gonzalez, and J. A. Pople, Gaussian, Inc., Pittsburgh, PA (1995), see also references therein for BPW91.

${ }^{34}$ L. D. Lloyd and R. L. Johnston, J. Chem. Phys. 236, 107 (1998).

${ }^{35}$ E. K. Parks, L. Zhu, J. Ho, and S. J. Riley, J. Chem. Phys. 100, 7206 (1994), 102, 7377 (1995); S. K. Nayak, S. N. Khanna, B. K. Rao, and P. Jena, J. Phys. Chem. 101, 1072 (1997).

${ }^{36}$ E. C. Honea, A. Ogura, C. A. Murray, K. Raghavachari, W. O. Sprenger, M. F. Jarrold, and W. L. Brown, Nature (London) 366, 42 (1993).

${ }^{37}$ C. W. Bauschlicher, Jr., H. Partridge, S. R. Langhoff, P. R. Taylor, and S. P. Walch, J. Chem. Phys. 86, 7007 (1987).

${ }^{38}$ C. A. Stearns and F. J. Kohl, High. Temp. Sci. 5, 113 (1973).

${ }^{39}$ F. L. King and M. M. Ross, Chem. Phys. Lett. 164, 131 (1989); R. F. K. Herzog, W. P. Poschenreider, and F. G. Satkiewicz, Radiat. Eff. 18, 199 (1973).

${ }^{40}$ K. Fuke, S. Nonose, N. Kikuchi, and K. Kaya, Chem. Phys. Lett. 147, 479 (1988). 
${ }^{41}$ S. D. Mahanti, T. A. Kaplan, and M. Barma, J. Appl. Phys. 49, 2084 (1978).

${ }^{42}$ P. Jena, B. K. Rao, and R. M. Nieminen, Solid State Commun. 59, 509 (1986); B. K. Rao, P. Jena, M. Manninen, and R. M. S. Nieminen, Phys. Rev. Lett. 58, 1188 (1987).
${ }^{43}$ C. Brèchignac, Ph. Cahuzac, F. Carlier, and M. de Frutos, Phys. Rev. Lett. 64, 2893 (1990).

${ }^{44}$ B. K. Rao, S. N. Khanna, and P. Jena, Phys. Rev. B (to be published).

${ }^{45}$ A. Nakajima, K. Hoshino, T. Naganuma, Y. Sone, and K. Kaya, J. Chem. Phys. 95, 7061 (1991). 